\title{
Search for heavy neutral Higgs bosons in two-doublet models
}

\author{
DELPHI Collaboration
}

\begin{abstract}
A search for pair production of neutral heavy Higgs bosons decaying into $b \bar{b}$ has been carried out in a study of hadronic decays of the $\mathrm{Z}$ boson into four jet final states using data taken by DELPHI in 1991 and 1992.

The two production mechanisms present in the two Higgs doublets scheme, bremsstrahlung production of $\mathrm{hZ}^{*}$ and associated production of $\mathrm{hA}$, may lead to four beauty jets well recognizable using the precise microvertex detector measurements. No evidence for a signal was found, leading to limits on $\mathrm{BR}(\mathrm{Z} \rightarrow$ $\mathrm{hA} \rightarrow 4 \mathrm{~b}$ ) from 3.5 to $5.5 \times 10^{-4}$ at $95 \%$ confidence level, depending on the mass of the ligthest Higgs. When combined with the results of the recent DELPHI standard Higgs search, this result allows the kinematical limit to be reached for the masses of $h$ and $A$ in the minimal supersymmetric extension of the Standard Model (MSSM) scheme. It also allows the $\tan \beta \leq 1$ domain to be explored, and a region above the kinematic limit for direct hA production is constrained by considering virtual hA production. Results are also given in the general two-doublet scheme.
\end{abstract}


P.Abreu ${ }^{21}$, W.Adam ${ }^{8}$, T.Adye ${ }^{38}$, E.Agasi ${ }^{31}$, I.Ajinenko ${ }^{43}$, R.Aleksan ${ }^{40}$, G.D.Alekseev ${ }^{15}$, P.P.Allport ${ }^{22}$, S.Almehed $^{24}$, F.M.L.Almeida ${ }^{48}$, S.J.Alvsvaag ${ }^{4}$, U.Amaldi ${ }^{8}$, S.Amato ${ }^{48}$, A.Andreazza ${ }^{28}$, M.L.Andrieux ${ }^{13}$, P.Antilogus $^{25}$, W-D.Apel ${ }^{16}$, Y.Arnoud ${ }^{40}$, B.Asman ${ }^{45}$, J-E.Augustin ${ }^{19}$, A.Augustinus ${ }^{31}, \quad$ P.Baillon ${ }^{8}$, P.Bambade $^{19}$, F.Barao ${ }^{21}$, R.Barate ${ }^{13}$, G.Barbiellini ${ }^{47}$, D.Y.Bardin ${ }^{15}$, G.J.Barker ${ }^{35}$, A.Baroncelli ${ }^{41}$, O.Barring ${ }^{8}$, J.A.Barrio $^{26}$,W.Bartl ${ }^{51}$, M.J.Bates ${ }^{38}$, M.Battaglia ${ }^{14}$, M.Baubillier ${ }^{23}$, J.Baudot ${ }^{40}$, K-H.Becks ${ }^{53}$, M.Begalli ${ }^{37}$, P.Beilliere $^{7}$, P.Beltran ${ }^{10}$, A.C.Benvenuti ${ }^{5}$, M.Berggren ${ }^{42}$, D.Bertrand ${ }^{2}$, F.Bianchi ${ }^{46}$, M.Bigi $^{46}$, M.S.Bilenky ${ }^{15}$, P.Billoir $^{23}$, J.Bjarne ${ }^{24}$, D.Bloch ${ }^{9}$, M.Blume ${ }^{53}$, S.Blyth ${ }^{35}$, V.Bocci ${ }^{39}$, T.Bolognese ${ }^{40}$, M.Bonesini $^{28}$, W.Bonivento $^{28}$, P.S.L.Booth ${ }^{22}$, G.Borisov ${ }^{43}$, C.Bosio ${ }^{41}$, B.Bostjancic ${ }^{44}$, S.Bosworth ${ }^{35}$, O.Botner ${ }^{49}$, B.Bouquet $^{19}$, C.Bourdarios ${ }^{19}$, T.J.V.Bowcock ${ }^{22}$, M.Bozzo ${ }^{12}$, P.Branchini ${ }^{41}$, K.D.Brand ${ }^{36}$, R.A.Brenner ${ }^{14}$, H.Briand ${ }^{23}$, C.Bricman ${ }^{2}$, L.Brillault ${ }^{23}$, R.C.A.Brown ${ }^{8}$, P.Bruckman ${ }^{17}$, J-M.Brunet ${ }^{7}$, L.Bugge ${ }^{33}$, T.Buran ${ }^{33}$, A.Buys ${ }^{8}, \quad$ M.Caccia $^{28}$, M.Calvi ${ }^{28}$, A.J.Camacho Rozas ${ }^{42}$, T.Camporesi ${ }^{8}$, V.Canale ${ }^{39}$, M.Canepa ${ }^{12}$, K.Cankocak ${ }^{45}$, F.Cao ${ }^{2}$, F.Carena ${ }^{8}$, P.Carrilho ${ }^{48}$, L.Carroll ${ }^{22}$, C.Caso ${ }^{12}$, V.Cassio ${ }^{46}$, M.V.Castillo Gimenez ${ }^{50}$, A.Cattai $^{8}$, F.R.Cavallo ${ }^{5}$, L.Cerrito ${ }^{39}$, V.Chabaud ${ }^{8}$, A.Chan $^{1}$, Ph.Charpentier ${ }^{8}$, L.Chaussard ${ }^{25}$, J.Chauveau ${ }^{23}$, P.Checchia $^{36}$, G.A.Chelkov ${ }^{15}$, P.Chliapnikov ${ }^{43}$, P.Chochula ${ }^{6}$, V.Chorowicz ${ }^{8}$, J.T.M.Chrin ${ }^{50}$, V.Cindro ${ }^{44}$, P.Collins $^{35}$, J.L.Contreras ${ }^{19}$, R.Contri ${ }^{12}$, E.Cortina ${ }^{50}$, G.Cosme ${ }^{19}$, F.Cossutti ${ }^{47}$, H.B.Crawley ${ }^{1}$, D.Crennell ${ }^{38}$, G.Crosetti $^{12}$, J.Cuevas Maestro ${ }^{34}$, S.Czellar ${ }^{14}$, E.Dahl-Jensen ${ }^{29}$, J.Dahm ${ }^{53}$, B.Dalmagne ${ }^{19}$, M.Dam ${ }^{33}$, G.Damgaard $^{29}$, A.Daum ${ }^{16}$, P.D.Dauncey ${ }^{38}$, M.Davenport ${ }^{8}$, W.Da Silva ${ }^{23}$, C.Defoix ${ }^{7}$, G.Della Ricca ${ }^{47}$, P.Delpierre $^{27}$, N.Demaria ${ }^{35}$, A.De Angelis ${ }^{8}$, H.De Boeck ${ }^{2}$, W.De Boer ${ }^{16}$, S.De Brabandere ${ }^{2}$, C.De Clercq ${ }^{2}$, M.D.M.De Fez Laso ${ }^{50}$, C.De La Vaissiere ${ }^{23}$, B.De Lotto ${ }^{47}$, A.De Min ${ }^{28}$, L.De Paula ${ }^{48}$, C.De Saint-Jean ${ }^{40}$, H.Dijkstra ${ }^{8}$, L.Di Ciaccio ${ }^{39}$, F.Djama ${ }^{9}$, J.Dolbeau ${ }^{7}$, M.Donszelmann ${ }^{8}$, K.Doroba ${ }^{52}$, M.Dracos ${ }^{9}$, J.Drees ${ }^{53}$, K.-A.Drees ${ }^{53}$, M.Dris ${ }^{32}$, Y.Dufour ${ }^{7}$, F.Dupont ${ }^{13}$, D.Edsall ${ }^{1}$, R.Ehret ${ }^{16}$, T.Ekelof ${ }^{49}$, G.Ekspong ${ }^{45}$, M.Elsing ${ }^{53}$, J-P.Engel $^{9}$, N.Ershaidat ${ }^{23}$, M.Espirito Santo ${ }^{21}$, V.Falaleev ${ }^{43}$, D.Fassouliotis ${ }^{32}$, M.Feindt ${ }^{8}$, A.Fenyuk ${ }^{43}$, A.Ferrer $^{50}$, T.A.Filippas ${ }^{32}$, A.Firestone ${ }^{1}$, H.Foeth ${ }^{8}$, E.Fokitis ${ }^{32}$, F.Fontanelli ${ }^{12}$, F.Formenti ${ }^{8}$, J-L.Fousset $^{27}$, B.Franek $^{38}$, P.Frenkiel ${ }^{7}$, D.C.Fries ${ }^{16}$, A.G.Frodesen ${ }^{4}$, R.Fruhwirth ${ }^{51}$, F.Fulda-Quenzer ${ }^{19}$, H.Furstenau ${ }^{8}$, J.Fuster $^{8}$, D.Gamba ${ }^{46}$, M.Gandelman ${ }^{18}$, C.Garcia $^{50}$, J.Garcia ${ }^{42}$, C.Gaspar ${ }^{8}$, U.Gasparini ${ }^{36}$, Ph.Gavillet ${ }^{8}$, E.N.Gazis ${ }^{32}$, D.Gele ${ }^{9}$, J-P.Gerber ${ }^{9}$, L.Gerdyukov ${ }^{43}, \quad$ D.Gillespie ${ }^{8}$, R.Gokieli ${ }^{52}, \quad$ B.Golob $^{44}$, J.J.Gomez Y Cadenas ${ }^{8}$, G.Gopal ${ }^{38}$, L.Gorn ${ }^{1}$, M.Gorski $^{52}$, V.Gracco ${ }^{12}$, F.Grard ${ }^{2}$, E.Graziani ${ }^{41}$, G.Grosdidier $^{19}$, P.Gunnarsson ${ }^{45}$, J.Guy ${ }^{38}$, U.Haedinger ${ }^{16}$, F.Hahn ${ }^{53}$, M.Hahn ${ }^{16}$, S.Hahn ${ }^{53}$, S.Haider ${ }^{31}$, Z.Hajduk ${ }^{17}$, A.Hakansson $^{24}$, A.Hallgren ${ }^{49}$, K.Hamacher ${ }^{53}$, W.Hao ${ }^{31}$, F.J.Harris ${ }^{35}$, V.Hedberg ${ }^{24}$, R.Henriques ${ }^{21}$, J.J.Hernandez $^{50}$, J.A.Hernando ${ }^{50}$, P.Herquet ${ }^{2}$, H.Herr ${ }^{8}$, T.L.Hessing ${ }^{8}$, E.Higon ${ }^{50}$, H.J.Hilke ${ }^{8}$, T.S.Hill ${ }^{1}$, S-O.Holmgren ${ }^{45}$, P.J.Holt ${ }^{35}$, D.Holthuizen ${ }^{31}$, P.F.Honore ${ }^{7}$, M.Houlden ${ }^{22}$, J.Hrubec ${ }^{51}$, K.Huet ${ }^{2}$, K.Hultqvist ${ }^{45}$, P.Ioannou $^{3}$, J.N.Jackson ${ }^{22}$, R.Jacobsson ${ }^{45}$, P.Jalocha ${ }^{17}$, R.Janik ${ }^{6}$, G.Jarlskog ${ }^{24}$, P.Jarry ${ }^{40}$, B.Jean-Marie ${ }^{19}$, E.K.Johansson ${ }^{45}$, L.Jonsson ${ }^{24}$, P.Juillot ${ }^{9}$, M.Kaiser ${ }^{16}$, G.Kalmus ${ }^{38}$, F.Kapusta ${ }^{23}$, M.Karlsson ${ }^{45}$, E.Karvelas ${ }^{10}$, S.Katsanevas $^{3}$, E.C.Katsoufis ${ }^{32}$, R.Keranen ${ }^{14}$, B.A.Khomenko ${ }^{15}$, N.N.Khovanski ${ }^{15}$, B.King ${ }^{22}$, N.J.Kjaer ${ }^{29}$, H.Klein $^{8}$, A.Klovning ${ }^{4}$, P.Kluit ${ }^{31}$, J.H.Koehne ${ }^{16}$, B.Koene ${ }^{31}$, P.Kokkinias ${ }^{10}$, M.Koratzinos ${ }^{8}$, V.Kostioukhine ${ }^{43}$, C.Kourkoumelis ${ }^{3}$, O.Kouznetsov ${ }^{12}$, P.-H.Kramer ${ }^{53}$, M.Krammer ${ }^{51}$, C.Kreuter ${ }^{16}$, J.Krolikowski ${ }^{52}$, I.Kronkvist ${ }^{24}$, Z.Krumstein $^{15}$, W.Krupinski ${ }^{17}$, P.Kubinec ${ }^{6}$, W.Kucewicz ${ }^{17}$, K.Kulka ${ }^{49}$, K.Kurvinen ${ }^{14}$, C.Lacasta $^{50}$, $^{\text {, }}$, I.Laktineh $^{25}$, C.Lambropoulos ${ }^{10}$, J.W.Lamsa ${ }^{1}$, L.Lanceri ${ }^{47}$, D.W.Lane ${ }^{1}$, P.Langefeld ${ }^{53}$, V.Lapin ${ }^{43}$, I.Last ${ }^{22}$, J-P.Laugier $^{40}$, R.Lauhakangas ${ }^{14}$, G.Leder ${ }^{51}$, F.Ledroit ${ }^{13}$, V.Lefebure ${ }^{2}$, C.K.Legan ${ }^{1}$, R.Leitner ${ }^{30}$, Y.Lemoigne ${ }^{40}$, J.Lemonne $^{2}$, G.Lenzen ${ }^{53}$, V.Lepeltier ${ }^{19}$, J.M.Levy ${ }^{9}$, D.Liko ${ }^{51}$, R.Lindner ${ }^{53}$, A.Lipniacka ${ }^{19}$, I.Lippi ${ }^{36}$, B.Loerstad $^{24}$, M.Lokajicek ${ }^{11}$, J.G.Loken ${ }^{35}$, A.Lopez-Fernandez ${ }^{8}$, M.A.Lopez Aguera ${ }^{42}$, M.los ${ }^{31}$, D.Loukas ${ }^{10}$, J.J.Lozano ${ }^{50}$, P.Lutz ${ }^{40}$, L.Lyons ${ }^{35}$, G.Maehlum ${ }^{16}$, J.Maillard ${ }^{7}$, A.Maio ${ }^{21}$, A.Maltezos ${ }^{10}$, V.Malychev ${ }^{15}$, F.Mandl $^{51}$, J.Marco ${ }^{42}$, B.Marechal ${ }^{48}$, M.Margoni ${ }^{36}$, J-C.Marin ${ }^{8}$, C.Mariotti ${ }^{41}$, A.Markou ${ }^{10}$, T.Maron $^{53}$, C.Martinez-Rivero $^{42}$, F.Martinez-Vidal ${ }^{50}$, S.Marti i Garcia ${ }^{50}$, F.Matorras ${ }^{42}$, C.Matteuzzi ${ }^{28}$, G.Matthiae ${ }^{39}$, M.Mazzucato $^{36}$, M.Mc Cubbin ${ }^{8}$, R.Mc Kay ${ }^{1}$, R.Mc Nulty ${ }^{22}$, J.Medbo ${ }^{49}$, C.Meroni ${ }^{28}$, W.T.Meyer ${ }^{1}$, M.Michelotto $^{36}$, E.Migliore ${ }^{46}$, I.Mikulec ${ }^{51}$, L.Mirabito ${ }^{25}$, U.Mjoernmark ${ }^{24}$, T.Moa ${ }^{45}$, R.Moeller ${ }^{29}$, K.Moenig ${ }^{8}$, M.R.Monge $^{12}$, P.Morettini ${ }^{12}$, H.Mueller ${ }^{16}$, W.J.Murray ${ }^{38}$, B.Muryn ${ }^{17}$, G.Myatt ${ }^{35}$, F.Naraghi ${ }^{13}$, F.L.Navarria ${ }^{5}$, S.Navas $^{50}$, P.Negri ${ }^{28}$, S.Nemecek ${ }^{11}$, W.Neumann ${ }^{53}$, N.Neumeister ${ }^{51}$, R.Nicolaidou ${ }^{3}$, B.S.Nielsen ${ }^{29}$, V.Nikolaenko ${ }^{25}$, P.Niss ${ }^{45}$, A.Nomerotski ${ }^{36}$, A.Normand ${ }^{35}$, W.Oberschulte-Beckmann ${ }^{16}$, V.Obraztsov $^{43}$, A.G.Olshevski ${ }^{15}$, R.Orava ${ }^{14}$, K.Osterberg ${ }^{14}$, A.Ouraou ${ }^{40}$, P.Paganini ${ }^{19}$, M.Paganoni ${ }^{28}$, P.Pages ${ }^{9}$, R.Pain ${ }^{23}$, H.Palka ${ }^{17}$, Th.D.Papadopoulou ${ }^{32}$, L.Pape ${ }^{8}$, F.Parodi ${ }^{12}$, A.Passeri ${ }^{41}$, M.Pegoraro ${ }^{36}$, J.Pennanen ${ }^{14}$, L.Peralta $^{21}$, V.Perevozchikov $^{43}$, H.Pernegger ${ }^{51}$, M.Pernicka ${ }^{51}$, A.Perrotta ${ }^{5}$, C.Petridou ${ }^{47}$, A.Petrolini ${ }^{12}$, H.T.Phillips $^{38}$, G.Piana $^{12}$, F.Pierre ${ }^{40}$, M.Pimenta ${ }^{21}$, S.Plaszczynski ${ }^{19}$, O.Podobrin ${ }^{16}$, M.E.Pol ${ }^{18}$, G.Polok ${ }^{17}$, P.Poropat ${ }^{47}$, V.Pozdniakov ${ }^{15}$, M.Prest ${ }^{47}$, P.Privitera ${ }^{39}$, A.Pullia ${ }^{28}$, D.Radojicic ${ }^{35}$, S.Ragazzi ${ }^{28}$, H.Rahmani ${ }^{32}$, J.Rames ${ }^{11}$, P.N.Ratoff ${ }^{20}$, A.L.Read ${ }^{33}$, M.Reale ${ }^{53}$, P.Rebecchi ${ }^{19}$, N.G.Redaelli ${ }^{28}$, M.Regler ${ }^{51}$, D.Reid ${ }^{8}$, P.B.Renton ${ }^{35}$, L.K.Resvanis ${ }^{3}$, F.Richard ${ }^{19}$, J.Richardson ${ }^{22}$, J.Ridky ${ }^{11}$, G.Rinaudo ${ }^{46}$, I.Ripp ${ }^{40}$, A.Romero ${ }^{46}$, I.Roncagliolo ${ }^{12}$, P.Ronchese $^{36}$, L.Roos ${ }^{13}$, E.I.Rosenberg ${ }^{1}$, E.Rosso ${ }^{8}$, P.Roudeau ${ }^{19}$, T.Rovelli ${ }^{5}$, W.Ruckstuhl ${ }^{31}$, V.Ruhlmann-Kleider ${ }^{40}$, A.Ruiz ${ }^{42}$, H.Saarikko ${ }^{14}$, Y.Sacquin ${ }^{40}$, A.Sadovsky ${ }^{15}$, G.Sajot $^{13}$, J.Salt $^{50}$, J.Sanchez ${ }^{26}$, M.Sannino ${ }^{12}$, H.Schneider ${ }^{16}$, M.A.E.Schyns ${ }^{53}$, G.Sciolla ${ }^{46}$, F.Scuri ${ }^{47}$, Y.Sedykh ${ }^{15}$, A.M.Segar ${ }^{35}$, A.Seitz ${ }^{16}$, R.Sekulin ${ }^{38}$, R.C.Shellard ${ }^{37}$, I.Siccama ${ }^{31}$, P.Siegrist ${ }^{40}$, S.Simonetti ${ }^{40}$, F.Simonetto ${ }^{36}$, A.N.Sisakian ${ }^{15}$, B.Sitar ${ }^{6}$, T.B.Skaali ${ }^{33}$, G.Smadja ${ }^{25}$, O.Smirnova ${ }^{15}$, G.R.Smith ${ }^{38}$, R.Sosnowski ${ }^{52}$, D.Souza-Santos ${ }^{37}$, T.Spassov ${ }^{21}$, E.Spiriti $^{41}$, S.Squarcia ${ }^{12}$, H.Staeck ${ }^{53}$, C.Stanescu ${ }^{41}$, S.Stapnes ${ }^{33}$, I.Stavitski ${ }^{36}$, G.Stavropoulos ${ }^{10}$, K.Stepaniak ${ }^{52}$, F.Stichelbaut $^{8}$, A.Stocchi ${ }^{19}$, J.Strauss ${ }^{51}$, R.Strub ${ }^{9}$, B.Stugu ${ }^{4}$, M.Szczekowski ${ }^{52}$, M.Szeptycka ${ }^{52}$, T.Tabarelli ${ }^{28}$, 
O.Tchikilev ${ }^{43}$, G.E.Theodosiou ${ }^{10}$, A.Tilquin ${ }^{27}$, J.Timmermans ${ }^{31}$, L.G.Tkatchev ${ }^{15}$, T.Todorov ${ }^{9}$, D.Z.Toet ${ }^{31}$, A.Tomaradze $^{2}$, B.Tome ${ }^{21}$, E.Torassa ${ }^{46}$, L.Tortora ${ }^{41}$, G.Transtromer ${ }^{24}$, D.Treille ${ }^{8}$, W.Trischuk ${ }^{8}$, G.Tristram ${ }^{7}$, A.Trombini ${ }^{19}$, C.Troncon ${ }^{28}$, A.Tsirou ${ }^{8}$, M-L.Turluer ${ }^{40}$, T.Tuuva ${ }^{14}$, I.A.Tyapkin ${ }^{23}$, M.Tyndel ${ }^{38}$, S.Tzamarias $^{22}$, B.Ueberschaer $^{53}$, S.Ueberschaer ${ }^{53}$, O.Ullaland ${ }^{8}$, V.Uvarov ${ }^{43}$, G.Valenti ${ }^{5}$, E.Vallazza ${ }^{8}$, J.A.Valls Ferrer ${ }^{50}$, C.Vander Velde ${ }^{2}$, G.W.Van Apeldoorn ${ }^{31}$, P.Van Dam ${ }^{31}$, W.K.Van Doninck ${ }^{2}$, J.Van Eldik ${ }^{31}$, G.Vegni ${ }^{28}$, L.Ventura $^{36}$, W.Venus ${ }^{38}$, F.Verbeure ${ }^{2}$. M.Verlato ${ }^{36}$, L.S.Vertogradov ${ }^{15}$, D.Vilanova ${ }^{40}$, P.Vincent ${ }^{25}$. L.Vitale ${ }^{47}$, E.Vlasov $^{43}$, A.S.Vodopyanov ${ }^{15}$, M.Voutilainen ${ }^{14}$, V.Vrba ${ }^{11}$, H.Wahlen ${ }^{53}$, C.Walck ${ }^{45}$, F.Waldner $^{47}$, A.Wehr ${ }^{53}$, M.Weierstall $^{53}$, P.Weilhammer ${ }^{8}$, A.M.Wetherell ${ }^{8}$, D.Wicke ${ }^{53}$, J.H.Wickens ${ }^{2}$, M.Wielers ${ }^{16}$, G.R.Wilkinson $^{35}$, W.S.C.Williams ${ }^{35}$, M.Winter ${ }^{9}$, M.Witek $^{8}$, G.Wormser ${ }^{19}$, K.Woschnagg ${ }^{49}$, K.Yip ${ }^{35}$, O.Yushchenko ${ }^{43}$, F.Zach ${ }^{25}$, A.Zaitsev $^{43}$, A.Zalewska ${ }^{17}$, P.Zalewski ${ }^{52}$, D.Zavrtanik ${ }^{44}$, E.Zevgolatakos ${ }^{10}$, N.I.Zimin ${ }^{15}$, M.Zito ${ }^{40}$, D.Zontar ${ }^{44}$, R.Zuberi ${ }^{35}$, G.C.Zucchelli ${ }^{45}$, G.Zumerle ${ }^{36}$

\footnotetext{
${ }^{1}$ Ames Laboratory and Department of Physics, Iowa State University, Ames IA 50011, USA

${ }^{2}$ Physics Department, Univ. Instelling Antwerpen, Universiteitsplein 1, B-2610 Wilrijk, Belgium and IIHE, ULB-VUB, Pleinlaan 2, B-1050 Brussels, Belgium and Faculté des Sciences, Univ. de l'Etat Mons, Av. Maistriau 19, B-7000 Mons, Belgium

${ }^{3}$ Physics Laboratory, University of Athens, Solonos Str. 104, GR-10680 Athens, Greece ${ }^{4}$ Department of Physics, University of Bergen, Allégaten 55, N-5007 Bergen, Norway

${ }^{5}$ Dipartimento di Fisica, Università di Bologna and INFN, Via Irnerio 46, I-40126 Bologna, Italy

${ }^{6}$ Comenius University, Faculty of Mathematics and Physics, Mlynska Dolina, SK-84215 Bratislava, Slovakia

${ }^{7}$ Collège de France, Lab. de Physique Corpusculaire, IN2P3-CNRS, F-75231 Paris Cedex 05, France

${ }^{8}$ CERN, CH-1211 Geneva 23, Switzerland

${ }^{9}$ Centre de Recherche Nucléaire, IN2P3 - CNRS/ULP - BP20, F-67037 Strasbourg Cedex, France

${ }^{10}$ Institute of Nuclear Physics, N.C.S.R. Demokritos, P.O. Box 60228, GR-15310 Athens, Greece

${ }^{11}$ FZU, Inst. of Physics of the C.A.S. High Energy Physics Division, Na Slovance 2, 180 40, Praha 8, Czech Republic

${ }^{12}$ Dipartimento di Fisica, Università di Genova and INFN, Via Dodecaneso 33, I-16146 Genova, Italy

${ }^{13}$ Institut des Sciences Nucléaires, IN2P3-CNRS, Université de Grenoble 1, F-38026 Grenoble Cedex, France

${ }^{14}$ Research Institute for High Energy Physics, SEFT, P.O. Box 9, FIN-00014 Helsinki, Finland

${ }^{15}$ Joint Institute for Nuclear Research, Dubna, Head Post Office, P.O. Box 79, 101000 Moscow, Russian Federation

${ }^{16}$ Institut für Experimentelle Kernphysik, Universität Karlsruhe, Postfach 6980, D-76128 Karlsruhe, Germany

${ }^{17}$ High Energy Physics Laboratory, Institute of Nuclear Physics, Ul. Kawiory 26a, PL-30055 Krakow 30, Poland

${ }^{18}$ Centro Brasileiro de Pesquisas Físicas, rua Xavier Sigaud 150, BR-22290 Rio de Janeiro, Brazil

${ }^{19}$ Université de Paris-Sud, Lab. de l'Accélérateur Linéaire, IN2P3-CNRS, Bat 200, F-91405 Orsay Cedex, France

${ }^{20}$ School of Physics and Materials, University of Lancaster, Lancaster LA1 4YB, UK

${ }^{21}$ LIP, IST, FCUL - Av. Elias Garcia, $14-1^{\circ}$, P-1000 Lisboa Codex, Portugal

${ }^{22}$ Department of Physics, University of Liverpool, P.O. Box 147, Liverpool L69 3BX, UK

${ }^{23}$ LPNHE, IN2P3-CNRS, Universités Paris VI et VII, Tour 33 (RdC), 4 place Jussieu, F-75252 Paris Cedex 05, France

${ }^{24}$ Department of Physics, University of Lund, Sölvegatan 14, S-22363 Lund, Sweden

${ }^{25}$ Université Claude Bernard de Lyon, IPNL, IN2P3-CNRS, F-69622 Villeurbanne Cedex, France

${ }^{26}$ Universidad Complutense, Avda. Complutense s/n, E-28040 Madrid, Spain

${ }^{27}$ Univ. d'Aix - Marseille II - CPP, IN2P3-CNRS, F-13288 Marseille Cedex 09, France

${ }^{28}$ Dipartimento di Fisica, Università di Milano and INFN, Via Celoria 16, I-20133 Milan, Italy

${ }^{29}$ Niels Bohr Institute, Blegdamsvej 17, DK-2100 Copenhagen 0, Denmark

${ }^{30} \mathrm{NC}$, Nuclear Centre of MFF, Charles University, Areal MFF, V Holesovickach 2, 180 00, Praha 8, Czech Republic

${ }^{31}$ NIKHEF-H, Postbus 41882, NL-1009 DB Amsterdam, The Netherlands

${ }^{32}$ National Technical University, Physics Department, Zografou Campus, GR-15773 Athens, Greece

${ }^{33}$ Physics Department, University of Oslo, Blindern, N-1000 Oslo 3, Norway

${ }^{34}$ Dpto. Fisica, Univ. Oviedo, C/P. Pérez Casas, S/N-33006 Oviedo, Spain

${ }^{35}$ Department of Physics, University of Oxford, Keble Road, Oxford OX1 3RH, UK

${ }^{36}$ Dipartimento di Fisica, Università di Padova and INFN, Via Marzolo 8, I-35131 Padua, Italy

${ }^{37}$ Depto. de Fisica, Pontificia Univ. Católica, C.P. 38071 RJ-22453 Rio de Janeiro, Brazil

${ }^{38}$ Rutherford Appleton Laboratory, Chilton, Didcot OX11 OQX, UK

${ }^{39}$ Dipartimento di Fisica, Università di Roma II and INFN, Tor Vergata, I-00173 Rome, Italy

${ }^{40}$ Centre d'Etude de Saclay, DSM/DAPNIA, F-91191 Gif-sur-Yvette Cedex, France

${ }^{41}$ Istituto Superiore di Sanità, Ist. Naz. di Fisica Nucl. (INFN), Viale Regina Elena 299, I-00161 Rome, Italy

${ }^{42}$ C.E.A.F.M., C.S.I.C. - Univ. Cantabria, Avda. los Castros, S/N-39006 Santander, Spain, (CICYT-AEN93-0832)

${ }^{43}$ Inst. for High Energy Physics, Serpukov P.O. Box 35, Protvino, (Moscow Region), Russian Federation

${ }^{44} \mathrm{~J}$. Stefan Institute and Department of Physics, University of Ljubljana, Jamova 39, SI-61000 Ljubljana, Slovenia

${ }^{45}$ Fysikum, Stockholm University, Box 6730, S-113 85 Stockholm, Sweden

${ }^{46}$ Dipartimento di Fisica Sperimentale, Università di Torino and INFN, Via P. Giuria 1, I-10125 Turin, Italy

${ }^{47}$ Dipartimento di Fisica, Università di Trieste and INFN, Via A. Valerio 2, I-34127 Trieste, Italy and Istituto di Fisica, Università di Udine, I-33100 Udine, Italy

${ }^{48}$ Univ. Federal do Rio de Janeiro, C.P. 68528 Cidade Univ., Ilha do Fundão BR-21945-970 Rio de Janeiro, Brazil

${ }^{49}$ Department of Radiation Sciences, University of Uppsala, P.O. Box 535, S-751 21 Uppsala, Sweden

${ }^{50}$ IFIC, Valencia-CSIC, and D.F.A.M.N., U. de Valencia, Avda. Dr. Moliner 50, E-46100 Burjassot (Valencia), Spain

${ }^{51}$ Institut für Hochenergiephysik, Österr. Akad. d. Wissensch., Nikolsdorfergasse 18, A-1050 Vienna, Austria

${ }^{52}$ Inst. Nuclear Studies and University of Warsaw, Ul. Hoza 69, PL-00681 Warsaw, Poland

${ }^{53}$ Fachbereich Physik, University of Wuppertal, Postfach 100 127, D-42097 Wuppertal 1, Germany
} 


\section{Introduction}

The most natural extension to the Higgs sector of the Standard Model (SM) is to add a second Higgs bosons doublet. Of the eight degrees of freedom contained in the two doublets, three represent Goldstone bosons which, through the Higgs mechanism of spontaneous symmetry breaking, give masses to the $\mathrm{W}^{ \pm}$and $\mathrm{Z}$, leaving five physical Higgs bosons $\left(\mathrm{H}^{+}, \mathrm{H}^{-}, \mathrm{h}^{0}, \mathrm{H}^{0}, \mathrm{~A}^{0}\right)$. There are two CP-even scalars $\mathrm{h}^{0}$ and $\mathrm{H}^{0}$, with a mixing angle $\alpha$, and one CP-odd pseudoscalar $\mathrm{A}^{0}$. At tree level the model is specified by six parameters: four Higgs bosons masses, the mixing angle $\alpha$ and $\tan \beta=v_{2} / v_{1}$, the ratio of the vacuum expectation values of the two doublets. While the decay of the $\mathrm{Z}$ into a pair of identical Higgs bosons is forbidden by Bose-Einstein statistics, the decay to $h^{0}$ and $\mathrm{A}^{0}$ is allowed. In fact, the decay modes $\mathrm{Z} \rightarrow \mathrm{hA}$ and $\mathrm{Z} \rightarrow \mathrm{hZ} \mathrm{Z}^{*}$ are complementary in the two-doublet model: if one decay mode is suppressed by mixing, the other is enhanced.

Supersymmetry is one of the most promising theoretical ideas for solving the naturalness and hierarchy problems of the Standard Model [1]. Its simplest implementation, the minimal supersymmetric extension of the Standard Model (MSSM), is a particular case of a two Higgs bosons doublets model. One doublet couples only to down-type quarks and charged leptons while the other couples to up-type quarks. The number of free parameters at tree level is only two [1]. These can be chosen to be $\tan \beta$ and $\mathrm{m}_{A}$ (the mass of the neutral pseudoscalar). Higher order corrections introduce dependences on other parameters, mainly the top and top-squark masses [2] [3]. In contrast to the SM Higgs boson, the possible mass range for the lightest MSSM Higgs boson is quite tightly constrained: $\mathrm{m}_{h} \lesssim 140 \mathrm{GeV} / \mathrm{c}^{2}[2][3]$. In addition, it is possible that $\mathrm{m}_{h}+\mathrm{m}_{A}<\mathrm{m}_{Z}$.

The relevant formulae for the production and decay ratios of the lightest neutral Higgs bosons $h^{0}$ and $\mathrm{A}^{0}$ (from now on referred as $\mathrm{h}$ and $\mathrm{A}$ ) in the two-doublet and MSSM models can be found in section 5.1.

Up to now, Higgs boson searches have been carried out using primarily the missing energy or leptonic signatures [4] [5]. This is the case for the SM Higgs boson search in the $\mathrm{hZ}^{*}$ mode, as well as the hA channel in the MSSM [1] where the best limits for heavy $\mathrm{h}$ and $\mathrm{A}$ bosons are obtained assuming that one of them has decayed into tau pairs. Since it is a fundamental property of Higgs bosons that their coupling to other particles is proportional to the mass of that particle, the dominant decay mode of heavy h's and A's in is expected to be into $\mathrm{b} \overline{\mathrm{b}}$ while the branching ratio into $\tau^{+} \tau^{-}$is only a few per cent. The influence of other parameters of the model on the branching ratios will be discussed in section 5. As the detection of two isolated $\tau$ 's has a low efficiency if the Higgs bosons are heavy the limits on $\mathrm{BR}(\mathrm{Z} \rightarrow \mathrm{hA})$ achieved so far do not go below the $10^{-3}$ level.

Exploring the dominant decay mode requires a search for events with four jets containing beauty particles. Since this final state is purely hadronic, the QCD background is very important, while the signal is expected to be small. This requires a high selectivity while keeping a reasonable efficiency for the Higgs channel. Since lighter Higgs bosons have already been excluded, the interesting high mass Higgs boson decays into $\mathrm{b} \overline{\mathrm{b}}$ will produce well separated jets. Therefore, the needed selectivity can be achieved by applying an efficient b-tagging method to events with at least four well separated jets. It should be noted that an irreducible Standard Model background due to the production of four b quarks in QCD processes is expected to be at the level of $10^{-3}$ relative to the production of two $b$ quarks.

If kinematically allowed, $\mathrm{h} \rightarrow 2 \mathrm{~A}$ may become the dominant decay mode of $\mathrm{h}$, giving rise to topologies different to those discussed above. This special case is analyzed in section 5.3. 
The combination of the hA search with the SM Higgs boson search, which covers the channel $\mathrm{hZ}^{*}$, allows certain regions in the $\left(\mathrm{m}_{h}, \mathrm{~m}_{A}\right)$ plane to be completely excluded. Within the MSSM, the increase in sensitivity for the hA channel translates into a significant increase of the mass domain which can be explored. If $\tan \beta$ is very large, there exists the possibility to extend the present searches above the kinematical limit via $h A^{*}$ production. However the Yukawa mode, $\mathrm{Z} \rightarrow \mathrm{hb} \overline{\mathrm{b}}$, where an $\mathrm{h}$ is radiated from a $\mathrm{b}$ quark, remains inaccessible even for the highest allowed values of $\tan \beta$ [6]. If $\tan \beta$ is lower than unity (a case not favoured by the MSSM), the gain in sensitivity allows a wide domain of masses left uncovered by the previous searches [4] to be excluded.

In the general two-doublet scheme, the decay branching ratio of $\mathrm{A}$ to $\mathrm{b} \overline{\mathrm{b}}$ depends only on $\tan \beta$, whereas for $\mathrm{h}$ it depends also on the mixing angle $\alpha$. Therefore $\mathrm{h}$ may decouple from $\mathrm{b} \overline{\mathrm{b}}$, which is not the case for the $\mathrm{A}$. To cope with this possibility, special analyses were needed in both in the $\mathrm{hA}$ and $\mathrm{h} \mathrm{Z}^{*}$ channels.

The present analyses are limited by the requirement that both Higgs bosons should be heavy enough to decay to $b \bar{b}$ well above threshold. Due to this fact, in the general twodoublet case the region of the parameter space where the masses of both Higgs bosons

are below $15 \mathrm{GeV} / \mathrm{c}^{2}$ is not covered. In the MSSM case, previous results [4] are used to complement the present ones in this region.

\section{Event analysis}

\subsection{Apparatus}

A summary of the specific properties of the DELPHI detector [7] relevant to this study follows. Charged particle tracks were measured in four cylindrical tracking chambers aligned parallel to the electron beam direction and to that of the 1.2 Tesla magnetic field. These were: the microvertex detector (VD), described below, the Inner Detector (ID) at radii 12 to $28 \mathrm{~cm}$, the Time Projection Chamber, the main tracking device, covering radii 30 to $122 \mathrm{~cm}$ and the Outer Detector at radii 197 to $208 \mathrm{~cm}$. In addition, two planes of drift chambers aligned perpendicular to the beam axis (Forward Chambers A and B) tracked particles in the forward region, covering polar angles $10^{\circ}$ to $30^{\circ}$ and $150^{\circ}$ to $170^{\circ}$.

The VD consisted of two independent half-shells inserted between the beam pipe and the ID. Each half-shell contained three concentric layers of silicon microstrip detectors located at radii $6.3,9$ and $11 \mathrm{~cm}$ respectively. They measured the $\mathrm{r} \phi$ coordinate (where $\mathrm{r}$ is the radius and $\phi$ the azimuthal angle around the beam axis) and covered polar angles between $43^{\circ}$ and $137^{\circ}$. The average resolution on these $\mathrm{r} \phi$ measurements was $8 \mu \mathrm{m}$.

Electromagnetic energy was measured by the High density Projection Chamber (HPC) in the barrel and by the Forward ElectroMagnetic Calorimeter (FEMC) in the endcaps. The HPC had layers of lead and gas covering polar angles from $40^{\circ}$ to $140^{\circ}$. The FEMC has lead glass blocks covering polar angles $10^{\circ}$ to $36^{\circ}$ and $144^{\circ}$ to $170^{\circ}$.

\subsection{Event samples}

The analysis presented hereafter is based on the total statistics collected by the DELPHI experiment during the years 1991 and 1992.

The standard DELPHI analysis to select hadronic Z decays [8] was applied to the data, leaving a total of 950,000 events. The efficiency of this selection was calculated to be $(96.0 \pm 0.5) \%$. 
In order to estimate the background from known processes, a sample of about 1,350,000 hadronic decays of the $\mathrm{Z}$ was generated using JETSET 7.3 [9], processed through the full detector simulation program for DELPHI [10], and reconstructed using the same procedure as for the real data. A total of 1,286,000 of these events passed the hadronic selection criteria. In addition, three samples of $\mathrm{Z}$ decays to $\mathrm{hA}$ at different $\mathrm{m}_{A}$ and $\mathrm{m}_{h}$, were generated and passed through the same chain as the background simulation. The chosen masses were $\mathrm{m}_{A}\left(\mathrm{~m}_{h}\right)=40(40), 60(15)$, and $20(60) \mathrm{GeV} / \mathrm{c}^{2}$. In all samples, the A decayed into $\mathrm{b} \overline{\mathrm{b}}$, as did the $\mathrm{h}$ in the first two; in the last it decayed into $c \bar{c}$. These samples contained 2000 events each, all of which passed the hadronic selection criteria.

The masses in the first two samples were chosen to represent extreme conditions (equal and very different masses) in the region close to the limits of our sensitivity. The efficiency for intermediate masses was interpolated using a simplified simulation of the detector response.

For the third sample, $h$ decaying into $c \bar{c}$, the range of variation of both masses is small (see section 5.1). It was also found using the simplified simulation that the mass dependence of the efficiency was negligible. Thus only one point was generated.

\subsection{Identification of tracks originating from secondary vertices}

The initial step in b-tagging was to define a primary vertex. As a first approximation, a vertex was formed using all tracks from the event and including the average interaction point (calculated from a set of around 100 events recorded in the same running period). If the global $\chi^{2}$ of this vertex was unacceptably high, (probability of the $\chi^{2}$ lower than $1 \%)$ the track with the highest contribution was removed, and the fit redone. This was repeated until a consistent vertex was found. The precision achieved in the vertex position was $60 \mu \mathrm{m}$ along the horizontal direction in the plane normal to the beam. The determination of the vertical coordinate was dominated by the position of the beam spot; to take into account possible displacements of the beam during the run, its error was conservatively set to $40 \mu \mathrm{m}$.

The impact parameter of a particle was defined as the minimum distance between its track (projected on the plane perpendicular to the beam) and the reconstructed primary vertex. The sign of the impact parameter was taken to be positive if the projected track intersected the axis of the jet it belonged to after the point of closest approach (in the direction of the momentum), and negative otherwise. The jet reconstruction algorithm is described in section 3 below.

The impact parameter resolution in the simulation was degraded by approximately $10 \%$ to match that observed in data. This correction was calculated on generic hadronic events (those kept after the first preselection cited in 2.2) and mostly affects the central part of the impact parameter distribution. Figure 1a shows a comparison between simulation and data for the impact parameter distribution after this correction.

A track was said to have an offset if it was within the acceptance of the microvertex detector, had hits associated in at least 2 VD layers, and had a positive impact parameter smaller than $2 \mathrm{~mm}$ (to avoid including decay products of $\mathrm{K}_{\mathrm{S}}^{0}$, or photon conversions) and larger than 2.5 standard deviations (including the error due to the primary vertex reconstruction). Particles with momentum below $0.5 \mathrm{GeV} / \mathrm{c}$ were not considered. A probability cut was applied to eliminate particles with possible errors in the association between its reconstructed track and VD detector hits.

A satisfactory agreement was observed in the distribution of the number of offsets between data and simulation, as shown in figure $1 \mathrm{~b}$. While the general agreement is good 
a certain difference arises for large number of offsets. Note that the simulated sample is of a size comparable to the data sample, and hence the errors of the simulated values are similar to those indicated by the error bars on the data points.

\section{Search for $\mathrm{Z} \rightarrow \mathrm{hA}$}

The hA decay mode of the $\mathrm{Z}$ is assumed to produce at least four well separated jets, since low masses of the Higgs bosons have been excluded by previous searches. To ensure that the jets were separated, only spherical events were retained before jet-clustering was performed. The criterion to select such events was that the sum of the two Fox-Wolfram moments [11] $H_{2}$ and $H_{4}$ should be less than 0.6. In calculating these moments, only charged particles passing the quality criteria described in [8] and electromagnetic clusters of energy bigger than $0.5 \mathrm{GeV}$ were used. This cut selected $9.4 \%$ of the real events and $9.2 \%$ of the simulated ones, while retaining $92 \%$ of simulated hA events with both bosons decaying into $\mathrm{b} \overline{\mathrm{b}}$ for $\mathrm{m}_{h}=\mathrm{m}_{A}=40 \mathrm{GeV} / \mathrm{c}^{2}$. The latter efficiency drops if one of the bosons is lighter; e.g. to $77 \%$ for $\mathrm{m}_{h}=15 \mathrm{GeV} / \mathrm{c}^{2}$ and $\mathrm{m}_{A}=60 \mathrm{GeV} / \mathrm{c}^{2}$.

In the sample of events that were classified as spherical, the particles were grouped into jets using the JADE algorithm with $y_{c u t}=0.01$. Events with less than four jets were rejected while those retained were forced to have four jets by increasing the value of $y_{\text {cut }}$ until exactly four jets were left.

To exploit the fact that the $\mathrm{A}$, and in most cases also the $\mathrm{h}$, decay predominantly into $\mathrm{b} \overline{\mathrm{b}}$, a procedure to select beauty jets was then applied. The procedure was based on the fact that the long lifetime and high mass of beauty particles gives their decay products large positive impact parameters. In addition, the multiplicity in the beauty decay is large. Hence, the procedure used the presence of many tracks with offsets, either in the event as a whole, or in a number of jets as will be detailed later. This method is well known to provide a robust selection of beauty particles which is not very sensitive to various backgrounds such as wrong associations of hits in the VD to reconstructed tracks, strange particle decays, or photon conversions [12].

Given the tagging efficiencies $\epsilon_{Q C D}$ and $\epsilon_{h A}$ for background and signal, respectively, the limit on the cross-section for $h \mathrm{~A}$ production is proportional to $\sqrt{\epsilon_{Q C D}} / \epsilon_{h A}$, as long as the errors on the estimated number of background events are statistics dominated and can be assumed to be Gaussian. In the following analysis, it was verified that the various cuts minimize this ratio or at least keep it almost constant while significantly improving the purity of the sample.

\subsection{Selection of the candidates}

Two different analyses were performed on the preselected sample of four-jet events. In the first, the aim was to search for an excess of events with a four jet topology and at least one $b \bar{b}$ pair. This analysis is sensitive to final states $b \bar{b} b \bar{b}$ and $b \bar{b} c \bar{c}$ and therefore covers the scenario where $h$ decouples from $b \bar{b}$. It was required that the total number of offsets in the event should be bigger than four. The sample of events that passed this criterion is referred to as Sample $I$ in the following.

The second analysis searched more specifically for an excess of events with two b $\bar{b}$ pairs, corresponding to the final state where both Higgs bosons decay into $\mathrm{b} \overline{\mathrm{b}}$. The simplest approach is to require four jets with at least two offsets in each jet. However, the average tagging efficiency per jet is only approximately $20 \%$ due to limited solid angle coverage by the VD (the probability that at least one jet is outside the VD acceptance is 
about $75 \%$ in first approximation), and to the softness of the beauty particles in four jet events.

A looser selection was therefore applied, requiring two jets with at least two offsets each and a total of at least two offsets in the remaining two jets. The set of events that satisfied this criterion is referred to as Sample II.

\subsection{QCD backgrounds}

From standard QCD processes, events with $4 \mathrm{~b}$ quarks are expected to be produced in about $0.03 \%$ of the hadronic Z decays (this figure was extracted with the JETSET [9] event generator, using the Parton Shower model). Events with two b quarks and two $c$ quarks are seven times more abundant. These two components give rise to an irreducible background, which, however, had a minor impact on this analysis. The main background comes from $\mathrm{b} \bar{b}$ events with at least two additional jets produced by energetic gluons, the

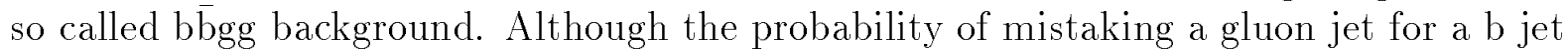
is small, this is compensated by the much larger cross section for this process.

According to the simulation, the main background in Sample I consists of b $\bar{b} g g$ events; a second heavy flavour ( $b$ or $c$ ) was present in only $10 \%$ of the cases. The fraction of events that did not contain a $\mathrm{b} \overline{\mathrm{b}}$ pair was $4 \%$.

In the simulated background classified as belonging to Sample II, 96\% of the events contained a $\mathrm{b} \overline{\mathrm{b}}$ pair; the rest had a $c \overline{\mathrm{c}}$ pair. In $13 \%$ of the cases a second $\mathrm{b} \overline{\mathrm{b}}$ pair was present, whereas $14 \%$ of the events had an additional $c \bar{c}$ pair.

\subsection{Systematic effects in the background estimation}

A detailed comparison between data and simulated events revealed a few significant discrepancies in the b-tagging efficiency and in the four jet selection.

The fraction of events (with any number of jets) with more than four offsets was around $10 \%$ higher in the simulated events than in the data. This effect comes from a slightly better track-microvertex association efficiency in the simulation, and small differences in the impact parameter resolution. The disagreement does not vary significantly when the number of demanded offsets increases from four to six. Therefore an average correction factor of 0.9 was applied to the selection efficiency of both samples for the simulated events, with an associated systematic uncertainty of \pm 0.05 .

It was also found that the number of events classified as four jets after the event preselection was $10 \%$ larger in the data than in the simulation. Detailed checks showed that this effect is uncorrelated with the b-tagging cuts, and hence that it affects both signal samples in the same way as the overall sample. The predicted number of background events of each sample was corrected and a systematic error of half of the correction, \pm $5 \%$, was assigned to it.

A further uncertainty arises from the JETSET prediction for $4 \mathrm{~b}$ final states which, so far, has never been experimentally tested. It is relevant mainly for Sample II. A $(13 \pm 4 \pm 5) \%$ contribution is expected in the final selection, where the first error comes from the statistics of simulated events and the second error from the uncertainty in their production rate. The assumed systematic errors are purely based on our confidence in the QCD calculation since the data do not yet allow such a precise determination.

Comparing the Matrix Element and Parton Shower options in the JETSET generator

[9] the differences observed were well within the quoted systematic error. It may be concluded that these estimates are conservative [13]. 
Other possible systematic effects such as those due to variation of the beauty lifetime and the effects of the cuts were found to be negligible.

All systematic errors were added in quadrature, giving a total contribution of $\pm 7 \%$ for Sample I and $\pm 10 \%$ for the Sample II.

\subsection{Results}

The efficiency for the hA channel was estimated using the simulation program for various values of $\mathrm{m}_{h}$ and $\mathrm{m}_{A}$. A lifetime of $1.6 \mathrm{ps}$ was assumed for all beauty particles except $\Lambda_{b}$, for which the lifetime was set to $1.1 \mathrm{ps}$.

The signal efficiency for Sample I (i.e. the sample aimed at the $\mathrm{b} \bar{b} c \bar{c}$ channel) was estimated to be $(8.0 \pm 0.5) \%$. In the range of $h$ and $A$ masses to which it applies, the result does not have a significant mass dependence. Taking into account the corrections discussed above and their associated systematic errors, $1956 \pm 38$ (stat) \pm 140 (syst) events were predicted and 1899 events found. No excess that could be due to Higgs boson production is found. This allows a limit to be set on the branching ratio for the channel $\mathrm{Z} \rightarrow \mathrm{h} A \rightarrow \mathrm{b} \overline{\mathrm{b}} \mathrm{c} \overline{\mathrm{c}}$ of $2.5 \times 10^{-3}$ relative to $\mathrm{Z} \rightarrow$ anything (at the $95 \%$ confidence level) in the region of interest (see the discussion in section 5.1 ): $\mathrm{m}_{h} \simeq 40-60 \mathrm{GeV} / \mathrm{c}^{2}$, $\mathrm{m}_{A} \simeq 20 \mathrm{GeV} / \mathrm{c}^{2}$.

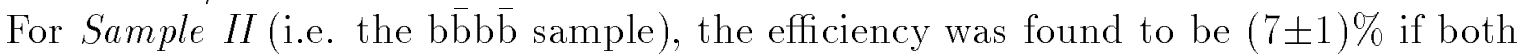
bosons had masses above $25 \mathrm{GeV} / \mathrm{c}^{2}$, and to increase smoothly to $(8.0 \pm 0.5) \%$ if they had masses above $35 \mathrm{GeV} / \mathrm{c}^{2}$. If one of the bosons had a mass of $15 \mathrm{GeV} / \mathrm{c}^{2}$, the efficiency was found to decrease to $(5.0 \pm 0.5) \%$. After correction for the systematic effects discussed above and taking into account the associated errors, $97 \pm 9$ (stat) \pm 10 (syst) events were predicted while 105 events were observed. It may therefore be concluded that at this level no significant excess due to Higgs boson production or other mechanisms is visible in the data. A corresponding limit on the branching ratio for $\mathrm{Z} \rightarrow \mathrm{h} A \rightarrow 4 \mathrm{~b}$ of $3.5 \times 10^{-4}$ relative to $\mathrm{Z} \rightarrow$ anything was derived at $95 \%$ confidence level for the case when both bosons' masses are above $35 \mathrm{GeV} / \mathrm{c}^{2}$. The limit increases to $5.5 \times 10^{-4}$ when one of the Higgs bosons has a mass of $15 \mathrm{GeV} / \mathrm{c}^{2}$. To compute the limits quoted above, the systematic and statistical errors where assumed to be Gaussian and added in quadrature. This is justified by the very conservative estimation of the systematic errors. The efficiency used in the calculation was the one given in the text minus its error, to account for the effect of its uncertainty. Furthermore, when the expected background was higher than the observed one (as in Sample I) a zero excess was taken.

Among the 105 selected candidates, one had four jets tagged, i.e. with at least 2 offsets in each jet. This event is shown in figures 2 and 3.

\section{Search for $\mathrm{Z} \rightarrow \mathrm{hZ} *$}

A general analysis of the two-doublet Higgs bosons model requires the two processes $\mathrm{Z} \rightarrow \mathrm{hA}$ and $\mathrm{Z} \rightarrow \mathrm{hZ}^{*}$ to be studied. For the latter, the results of the Standard Model Higgs boson search described in [14] were used. Since the analysis described in [14] partly relies on b-tagging, it was necessary to perform an additional analysis in order to calculate limits to be applied in the regions where the $h$ may decouple from $b \bar{b}$. The initial selection of the neural network method used in [14] for the analysis of the h $\nu \bar{\nu}$ channel does not depend on the b-tagging information; this is used only in the independent cuts applied as a second step. Therefore it is straightforward to remove the b-tagging cuts. When this is done, two candidates are accepted in the final selection with visible masses $(50 \pm 7) \mathrm{GeV} / \mathrm{c}^{2}$ 
and $(45 \pm 7) \mathrm{GeV} / \mathrm{c}^{2}$ respectively. The first candidate has missing momentum pointing at a polar angle of $90^{\circ}$, where the HPC is insensitive, but where two lead-scintillator veto counters show a significant activity. This event can therefore be removed as a probable $q \bar{q} \gamma$ background, where the photon is missed by the electromagnetic calorimeter, with negligible effect on the efficiency for the signal. The second candidate is retained.

In section 5.1, the limit on hZ* without b-tag is essential for masses above $45 \mathrm{GeV} / \mathrm{c}^{2}$ and therefore the remaining candidate at $45 \pm 7 \mathrm{GeV} / \mathrm{c}^{2}$ has a significant influence on the two-doublet limit. Also, the h $\nu \bar{\nu}$ limit is weaker than that obtained in [14] where the neural network method was combined with a probabilistic method to increase the efficiency by about $15 \%$.

\section{Application to Higgs bosons searches}

In this section, the analysis presented in [4] is extended, taking into account the recent progress achieved in the hZ* analysis and the present result on $4 \mathrm{~b}$ final states for $\mathrm{hA}$.

\subsection{Two-doublet limits}

In the two-doublet scheme, the branching ratios of $\mathrm{Z}$ to $\mathrm{hA}$ and $\mathrm{h} \mathrm{Z}^{*}$ depend on mixing effects. Defining the Standard Model width for $h Z^{*}$ by $\Gamma_{\mathrm{SM}}\left(\mathrm{Z} \rightarrow \mathrm{h} \mathrm{Z}^{*}\right)$, one has:

$$
\begin{aligned}
\Gamma\left(\mathrm{Z} \rightarrow \mathrm{h} \mathrm{Z}^{*}\right) & =\Gamma_{\mathrm{SM}}\left(\mathrm{Z} \rightarrow \mathrm{h} \mathrm{Z}^{*}\right) \sin ^{2}(\alpha-\beta) \\
\Gamma(\mathrm{Z} \rightarrow \mathrm{hA}) & =0.5 \Gamma(\mathrm{Z} \rightarrow \nu \bar{\nu}) \lambda^{3} \cos ^{2}(\alpha-\beta)
\end{aligned}
$$

where $\alpha$ and $\beta$ are the mixing angles in the two-doublet scheme, while $\lambda^{3}$ is a phase space factor (see appendix). These expressions clearly indicate the complementarity of the two processes. If one of them is experimentally out of reach for a given set of masses $\mathrm{m}_{h}$ and $\mathrm{m}_{A}$, no exclusion is possible since mixing angles could always be such that the other process is suppressed below detectability. The limits on each channel can be translated into limits on $\cos ^{2}(\alpha-\beta)$ and $\sin ^{2}(\alpha-\beta)$ and hence to a limit on $\cos ^{2}(\alpha-\beta)+\sin ^{2}(\alpha-\beta)$. A given $\mathrm{m}_{h}$ and $\mathrm{m}_{A}$ combination can be excluded if this last limit is less than unity.

For $\tan \beta \geq 1$, A always decays dominantly into b quarks, while the decay rates of $h$ depend on the mixing-angle $\alpha[1]$ :

$$
\begin{aligned}
\Gamma(\mathrm{A} \rightarrow \mathrm{b} \overline{\mathrm{b}}) & \sim \mathrm{m}_{\mathrm{b}}^{2} \tan ^{2} \beta \\
\Gamma(\mathrm{A} \rightarrow \mathrm{c} \overline{\mathrm{c}}) & \sim \mathrm{m}_{\mathrm{c}}^{2} / \tan ^{2} \beta \\
\Gamma(\mathrm{h} \rightarrow \mathrm{b} \overline{\mathrm{b}}) & \sim \mathrm{m}_{\mathrm{b}}^{2} \sin ^{2} \alpha / \cos ^{2} \beta \\
\Gamma(\mathrm{h} \rightarrow \mathrm{c} \overline{\mathrm{c}}) & \sim \mathrm{m}_{\mathrm{c}}^{2} \cos ^{2} \alpha / \sin ^{2} \beta
\end{aligned}
$$

where $-\alpha$ varies between 0 and $\pi / 2$. For $\alpha=0$, h decouples from $b \bar{b}$ and decays into $c \bar{c}$ (the decay fraction to $\tau^{+} \tau^{-}$is negligible). Hence, in this case $\mathrm{hA}$ will decay into $\mathrm{b} \overline{\mathrm{b}} \mathrm{c} \overline{\mathrm{c}}$ final states, detectable in Sample I. The corresponding limit on $\operatorname{BR}(\mathrm{Z} \rightarrow \mathrm{b} \overline{\mathrm{b}} c \overline{\mathrm{c}})$ is, on average, $2.5 \times 10^{-3}$. As mentioned in section 4 , the $h Z^{*}$ analysis [14] can be performed without b-tagging.

The exclusion contours given in figure 4 are obtained for the choice of $\alpha$ and $\beta$ leading to the weakest limit at any given mass combination. Figure 4 a shows the limits obtained when $\tan \beta \geq 1$. For $h$ heavier than $47 \mathrm{GeV} / \mathrm{c}^{2}$ the limits on $\sin ^{2}(\alpha-\beta)$ given by the search for $h Z^{*}$ are above 0.5 , and therefore the case $\alpha=0, \beta=\pi / 4$ is allowed. Thus, the decoupling of $\mathrm{h}$ from $\mathrm{b} \overline{\mathrm{b}}$ cannot be excluded and the searches assuming $h \rightarrow b \bar{b}$ are 
ineffective. For h lighter than $47 \mathrm{GeV} / \mathrm{c}^{2}, \alpha=0$ is excluded by the $\mathrm{hZ} \mathrm{Z}^{*}$ analysis in the case $h \rightarrow c \bar{c}$ and the allowed values for $\alpha$ and $\beta$ give large branching ratios for both $h$ and $\mathrm{A}$ into $b \bar{b}$. Hence the results obtained for $h \rightarrow b \bar{b}$ apply, resulting in more stringent limits. Thus the exclusion region shows a step at $\mathrm{m}_{h}=47 \mathrm{GeV} / \mathrm{c}^{2}$. The outcome is a very significant coverage of the heavy mass sector of $h$ and $A$. This is true even for $\tan \beta$ below 1 , as can be seen in figure $4 \mathrm{~b}$. Here again the step appearing in the limit, at $\mathrm{m}_{h}=38 \mathrm{GeV} / \mathrm{c}^{2}$, corresponds to the transition between the strong limit obtained when $\mathrm{h}$ has to decay into $\mathrm{b} \overline{\mathrm{b}}\left(\sin ^{2}(\alpha-\beta)<0.2\right.$, which would imply $\alpha>0$ when $\left.\tan \beta=0.5\right)$ and the weaker limit obtained when $h$ can be decoupled from $b \bar{b}$. There is a substantial improvement with respect to the previously published limits [4] based on the $\tau^{+} \tau^{-}$decays of the hor A.

\subsection{MSSM limits}

In the MSSM Higgs sector, which is a special case of the two-doublet scheme, the relative production and decay branching ratios of (1) and (2) apply also, but the allowed domain for $m_{h}$ and $m_{A}$ is restricted. Also, $\sin ^{2}(\alpha-\beta)$ and $\cos ^{2}(\alpha-\beta)$ are restricted to vary within a certain range for any given $\mathrm{m}_{A}$ and $\mathrm{m}_{h}$. In particular $\alpha$ does not vanish and hence $h$ always couples to $\mathrm{b} \bar{b}$. The relations between these parameters depend on the unknown parameters of the MSSM through radiative corrections. Reference [2] contains calculations of these corrections including one-loop effects. Using them and assuming degeneracy of the two top-squark masses, only the top and top-squark masses dependences remains. The exclusion region obtained is shown in figure 5. A top mass of $170 \mathrm{GeV} / \mathrm{c}^{2}$ (which agrees with the direct measurement of CDF [15] and that deduced from the precision measurements of LEP/SLC [16]), a top-squark mass of $1 \mathrm{TeV} / \mathrm{c}^{2}$ and $\tan \beta>1$ are assumed. To treat the region below $15 \mathrm{GeV}$ the $\mathrm{b} \overline{\mathrm{b}}$ threshold the results of [4] have been used.

It is unlikely that $\tan \beta \leq 1$, since in Grand Unified Theories this choice is incompatible with a correct description of electro-weak symmetry breaking [17]. The constraint that the Yukawa couplings do not develop a Landau-pole at high energies imposes $\tan \beta \geq 0.5$ [18]. However, the same procedure to determine limits can also be applied in this case. One finds that for $0.5<\tan \beta<1$, most of the domain accessible at LEP100 is unphysical and the present search constrains $\mathrm{m}_{h}$ to be above $55 \mathrm{GeV} / \mathrm{c}^{2}$. There is, however, no lower limit on $\mathrm{m}_{A}$ when $\mathrm{m}_{h} \geq 60 \mathrm{GeV} / \mathrm{c}^{2}$.

Hence, at $95 \%$ confidence level:

- $\mathrm{m}_{h} \geq 44 \mathrm{GeV} / \mathrm{c}^{2}$ for any $\tan \beta$

- $\mathrm{m}_{A} \geq 27 \mathrm{GeV} / \mathrm{c}^{2}$ for $\tan \beta \geq 1$

assuming that $\mathrm{m}_{t}=170 \mathrm{GeV} / \mathrm{c}^{2}$ and $\mathrm{m}_{\mathrm{sq}}=1 \mathrm{TeV} / \mathrm{c}^{2}$.

The $\mathrm{m}_{h}$ limit is almost insensitive to the top and top-squark masses. The variation of the limit on $\mathrm{m}_{A}$ as a function of these masses is shown in figure 6 . A significant dependence of the $m_{A}$ mass limit on the top-squark mass is observed above $550 \mathrm{GeV} / \mathrm{c}^{2}$, which becomes milder above $700 \mathrm{GeV} / \mathrm{c}^{2}$.

Figure 7 shows the same results in the representation $\tan \beta$ vs $\mathrm{m}_{A}$. The limit on $\mathrm{m}_{A}$ is seen to depend on the top mass only for $\tan \beta$ below 4 . It should be noted that if $\tan \beta \gtrsim$ 1.5, $\mathrm{m}_{A}$ is excluded up to $44 \mathrm{GeV} / \mathrm{c}^{2}$.

These results represent a significant improvement compared to our previous limits [4], in particular that on $\mathrm{m}_{A}$. The lowest limit on $\mathrm{m}_{A}\left(27 \mathrm{GeV} / \mathrm{c}^{2}\right)$ corresponds to $\tan \beta \simeq 1$ (as can be seen in figure $7 \mathrm{a}$ ) and $\mathrm{m}_{h} \simeq 58 \mathrm{GeV} / \mathrm{c}^{2}$ (see figure 5). It can be shown that 
in this region the dominant contribution to the limit comes from the $\mathrm{Z} \rightarrow \mathrm{h} \mathrm{Z}^{*}$ search. An increased sensitivity on this channel would lead to further progress on the $\mathrm{m}_{A}$ limit.

In a number of recent publications [3], the radiative corrections to the Higgs boson mass have been calculated including two-loop effects. Using these calculations, the limit on $\mathrm{m}_{A}$ becomes more stringent. With the same values for $\mathrm{m}_{t}$ and $\mathrm{m}_{s q}\left(170 \mathrm{GeV} / \mathrm{c}^{2}\right.$ and $1 \mathrm{TeV} / \mathrm{c}^{2}$, respectively), and assuming $\alpha_{s}=0.11$, the branching ratio limits reported here yield

- $\mathrm{m}_{h} \geq 44 \mathrm{GeV} / \mathrm{c}^{2}$ for any $\tan \beta$

- $\mathrm{m}_{A} \geq 39 \mathrm{GeV} / \mathrm{c}^{2}$ for $\tan \beta \geq 1$.

On-shell production of hA in $\mathrm{Z}$ decays is excluded in this case. Also $\tan \beta=1$ is excluded if $\mathrm{m}_{t}$ is below $150 \mathrm{GeV} / \mathrm{c}^{2}$. In this case the $\mathrm{m}_{h}$ limit is also insensitive to the top and top-squark masses, while the drop in the $\mathrm{m}_{A}$ limit appears at higher top-squark masses than in the case of one-loop radiative corrections; it is observed around $1 \mathrm{TeV} / \mathrm{c}^{2}$. For $0.5 \leq \tan \beta \leq 1, \mathrm{~m}_{h}$ must be above $52 \mathrm{GeV} / \mathrm{c}^{2}$; if it is above $63 \mathrm{GeV} / \mathrm{c}^{2}$, no limit can be set on $\mathrm{m}_{A}$.

\subsection{The $\mathrm{h} \rightarrow 2$ A decay mode}

In the two-doublet model the decay width of $\mathrm{h} \rightarrow \mathrm{AA}$ is simply related to that of $\mathrm{h} \rightarrow \mathrm{b} \overline{\mathrm{b}}$ through the expression [1]:

$$
\frac{\Gamma(\mathrm{h} \rightarrow \mathrm{AA})}{\Gamma(\mathrm{h} \rightarrow \mathrm{b} \overline{\mathrm{b}})}=\frac{1}{12} \frac{\beta_{A}}{\beta_{b}^{3}} \frac{m_{Z}^{4}}{m_{b}^{2} m_{h}^{2}} \frac{\cos ^{2}(2 \beta) \sin ^{2}(\beta+\alpha)}{\tan ^{2} \beta}
$$

with the usual meaning for $\beta_{A}$ and $\beta_{b}$. Except for particular values of the mixing angles: $\beta=\frac{\pi}{4}, \frac{\pi}{2}$ or $\alpha+\beta=0, \mathrm{~h} \rightarrow \mathrm{AA}$ largely dominates. In the case considered so far, when $\mathrm{A}$ is heavy and decays predominantly into $\mathrm{h} \rightarrow \mathrm{b} \overline{\mathrm{b}}$, the reaction $\mathrm{Z} \rightarrow \mathrm{hA}$ will give a final state containing six b quarks.

The efficiency for the $6 \mathrm{~b}$ final state has been calculated, using the full simulation, with the cuts leading to Sample II (see section 3.2). As expected, the b-tagging efficiency increases and the resulting overall efficiency is approximately twice as large as for the $4 \mathrm{~b}$ final state. For example with $\mathrm{m}_{A}=27 \mathrm{GeV} / \mathrm{c}^{2}$ and $\mathrm{m}_{h}=58 \mathrm{GeV} / \mathrm{c}^{2}$ it is $\epsilon_{6 b}=(17 \pm 2) \%$ while $\epsilon_{4 b}=(7 \pm 1) \%$. Therefore, taking into account this gain in efficiency, a significant improvement on the hA limits could be expected. However it turns out that, as explained below, the mass limits are practically unchanged.

In the general two-doublet model, as long as $\tan \beta \geq 1$, when $\alpha=0$ is allowed by the $\mathrm{hZ}^{*}$ limit, $\beta$ can always be set to $\frac{\pi}{4}$ so that both the $\mathrm{b} \overline{\mathrm{b}}$ and the $2 \mathrm{~A}$ mode are absent. Since the mass limits are based on the least favourable decay mode, they do not change in this case. This does not hold when $\tan \beta$ falls below 1 , since $\beta$ can no more be fixed to $\frac{\pi}{4}$ to eliminate the $\mathrm{h} \rightarrow \mathrm{AA}$ mode. As a result, a small improvement on the mass limits of figure $4 \mathrm{~b}$ would be obtained for $\mathrm{m}_{h}>47 \mathrm{GeV} / \mathrm{c}^{2}$. However this effect is small and has been neglected.

In the MSSM case, most of the mass domain where $\mathrm{m}_{h}>2 \mathrm{~m}_{A}$ has already been excluded by the $4 \mathrm{~b}$ search. The limit on $\mathrm{m}_{A}$ at $27 \mathrm{GeV} / \mathrm{c}^{2}$, which as can be seen in figure 5 corresponds to $\mathrm{m}_{h}=58 \mathrm{GeV} / \mathrm{c}^{2}$, could in principle change since at this point the mode $\mathrm{h} \rightarrow \mathrm{AA}$ is allowed. However, this point is at the limit of the unphysical region corresponding to $\tan \beta=1$. From equation 3 , it follows that $\Gamma(\mathrm{h} \rightarrow \mathrm{AA})=0$ and therefore the limit remains unchanged. 


\subsection{Comment on searches for $\mathrm{hA} *$}

If $\tan \beta$ is very large, the Yukawa couplings of $\mathrm{A}$ and $\mathrm{h}$ to $\mathrm{b} \overline{\mathrm{b}}$ are also large. This assumes that $\tan \alpha=-\tan \beta$, as predicted by the MSSM in the mass region considered here, as shown in the appendix. With such large couplings, it becomes possible to extend the present search above the kinematical limit either through the Yukawa mode, $h \mathrm{~b} \bar{b}$, or through virtual production of one of the Higgs bosons in the processes $e^{+} e^{-} \rightarrow \mathrm{h} \mathrm{A}^{*}$ or $e^{+} e^{-} \rightarrow h^{*} A$. In [6], it was shown that the Yukawa term is very small, while $h A^{*}$ could contribute to extend the limit somewhat beyond the kinematical limit of real hA production. Assuming $\mathrm{m}_{h}=\mathrm{m}_{A}$, and taking into account both $\mathrm{hA}^{*}$ and $\mathrm{h}^{*} \mathrm{~A}$, the branching ratio for the process (see appendix) is displayed in figure 8. This figure shows that only modest progress can be achieved beyond the kinematical limit. Even for a branching ratio limit of $10^{-4}$ and for $\tan \beta=40$ the limit on $\mathrm{m}_{h}\left(\mathrm{~m}_{A}\right)$ is still around $47 \mathrm{GeV} / \mathrm{c}^{2}$.

\section{Summary}

Beauty tagging using the DELPHI microvertex detector has set a stringent limit on the process $\mathrm{Z} \rightarrow \mathrm{hA} \rightarrow 4 \mathrm{~b}$ which, when combined with results from the latest DELPHI search for the SM Higgs boson, significantly restricts the allowed domain for neutral Higgs bosons masses.

For the two-doublet scheme, the mass limits obtained are close to the kinematical limit for the hA channel up to $\mathrm{m}_{h} \sim 47 \mathrm{GeV} / \mathrm{c}^{2}$ for $\tan \beta \geq 1$ and $\mathrm{m}_{h} \sim 38 \mathrm{GeV} / \mathrm{c}^{2}$ for $0.5 \leq \tan \beta<1$. For larger $\mathrm{m}_{h}$ a light $\mathrm{A}$ cannot be excluded.

In terms of the MSSM, the limits found are even more restrictive. The $\mathrm{h}$ mass limit of $44 \mathrm{GeV} / \mathrm{c}^{2}$ is obtained at $\mathrm{m}_{A}=45 \mathrm{GeV} / \mathrm{c}^{2}$, close to the kinematical limit for the $\mathrm{hA}$ channel, and is almost insensitive to the assumptions on the top and top-squark masses. The A mass limit depends on the theoretical formula used, and also on the top and top-squark masses. Assuming a top mass of $170 \mathrm{GeV} / \mathrm{c}^{2}$ and both top-squarks masses degenerate with a common value of $1 \mathrm{TeV} / \mathrm{c}^{2}$ this limit is about $27 \mathrm{GeV} / \mathrm{c}^{2}$ using radiative corrections calculated including one-loop effects, and $39 \mathrm{GeV} / \mathrm{c}^{2}$ if also two-loop effects are considered. These limits increase to $45 \mathrm{GeV} / \mathrm{c}^{2}$ if the top-squark mass is lower, below $550 \mathrm{GeV} / \mathrm{c}^{2}$ in the one-loop case, $900 \mathrm{GeV} / \mathrm{c}^{2}$ in the two-loop one. Further progress on the limit on $\mathrm{m}_{A}$ can be achieved by improving the sensitivity in the $\mathrm{h} \mathrm{Z}^{*}$ channel.

\section{Acknowledgements}

We are greatly indebted to our technical collaborators and to the funding agencies for their support in building and operating the DELPHI detector, and to the members of the CERN-SL Division for the excellent performance of the LEP collider. 


\section{APPENDIX}

In deriving figure 8 , it is assumed that $\tan \beta$ is large and that $A$ and $h$ have the same mass, just above the kinematical limit $\mathrm{m}_{Z} / 2$. The coupling constants of $\mathrm{h}$ and $\mathrm{A}$ to $\mathrm{b} \overline{\mathrm{b}}$ are proportional to $\sin \alpha / \cos \beta$ and $\tan \beta$ respectively. For large $\tan \beta$, it can be shown that:

$$
\tan \alpha=-\tan \beta \frac{m_{Z}^{2}+\epsilon-m_{A}^{2}}{m_{Z}^{2}+m_{A}^{2}}
$$

where $\epsilon$ is the loop correction term [2]. With a top mass of $170 \mathrm{GeV} / \mathrm{c}^{2}$, a top-squark mass of $1 \mathrm{TeV} / \mathrm{c}^{2}$ and a large $\tan \beta$, one finds $\epsilon=\left(76 \mathrm{GeV} / \mathrm{c}^{2}\right)^{2}$. Numerically, $\tan \alpha$ and $-\tan \beta$ are found to differ by less than $20 \%$ when $m_{A}$ is close to the kinematic limit.

The matrix element for the process $Z \rightarrow h A^{*}$ has been rederived and agrees with [6]. Assuming that $\mathrm{A}$ and $\mathrm{h}$ have the same mass and $\tan \beta$ is large, the final formula reads:

$$
\Gamma\left(h A^{*}+h^{*} A\right)=\frac{\alpha^{2} m_{b}^{2} \tan ^{2} \beta \cos ^{2}(\alpha-\beta)}{8 \pi m_{Z} \sin ^{4} \theta_{W} \cos ^{4} \theta_{W}} B W
$$

with

$$
B W=\int_{2 m_{b}}^{\left(m_{Z}-m_{h}\right)}\left|\bar{p}_{h}\right|^{3} \frac{\left(m / m_{Z}\right)^{3}}{\left(m^{2}-m_{A}^{2}\right)^{2}+\Gamma_{A}^{2} m_{A}^{2}} d m
$$

where the integration is over the mass of the virtual Higgs boson, and the momentum is in the centre-of-mass. It can be verified that with this formula the standard value of equation 1 is recovered when both $\mathrm{h}$ and $\mathrm{A}$ are on shell. The factor $\lambda^{3}$ used here, and in the rest of the paper, is the usual phase space factor:

$$
\lambda=\sqrt{\left(1-\frac{m_{h}^{2}}{m_{Z}^{2}}-\frac{m_{A}^{2}}{m_{Z}^{2}}\right)^{2}-4 \frac{m_{h}^{2}}{m_{Z}^{2}} \frac{m_{A}^{2}}{m_{Z}^{2}}}
$$




\section{References}

[1] See J.F. Gunion, H.E. Haber, G. Kane and S. Dawson, "The Higgs Hunters Guide", (Addison-Wesley, Reading, MA, 1990) and references therein.

[2] Y. Okada, M. Yamaguchi and T. Yanagida, Prog. Theor. Phys. Lett. 85 (1991) 1 ;

J. Ellis, G. Ridolfi and F. Zwirner, Phys. Lett. B 257 (1991) 83;

H.E. Haber and R. Hempfling, Phys. Rev. Lett. 66 (1991) 1815.

[3] J. Kodaira, Y. Yasui and K. Sasaki, Phys. Rev. D50 (1994) 7035;

R. Hempfling and A.H. Hoang, Phys. Lett. B 331 (1994) 99;

J.A. Casas, J.R. Espinosa, M. Quirós and A. Riotto, "The lightest Higgs boson mass in the Minimal Supersymmetric Standard Model"; preprint CERNTH.7334/94 (1994).

[4] DELPHI Collaboration, P. Abreu et al., Nucl. Phys. B 373 (1992) 3;

DELPHI Collaboration, P. Abreu et al., "Neutral Higgs Bosons in a Two doublet Model"; DELPHI Note 92-80 (June 1992). See also: G. Wormser, in "Proceedings of the Intl. Conf. on HEP Dallas, Texas"; J.R. Sanford, ed., AIP New York (1993), 1309-1314.

[5] ALEPH Collaboration, D. Buskulic et al., Phys. Lett. B 313 (1993) 312;

L3 Collaboration, O. Adriani et al., Z.Phys.C 57, 355-381(1993)

OPAL Collaboration, R. Akers et al., Z.Phys.C 64, 1-13(1994)

[6] J. Kalinowski and H.P. Nilles, Phys. Lett. B 255 (1991) 134.

[7] DELPHI Collaboration, P. Aarnio et al., Nucl. Instrum. Methods A303 (1991) 233.

[8] DELPHI Collaboration, P. Abreu et al., Nucl. Phys. B 417 (1994) 3.

[9] T. Sjöstrand, Comp. Phys. Comm. 39 (1986) 347.

[10] DELPHI Collaboration, "DELPHI event generation and detector simulation - User guide", DELPHI Note 89-67 (1989), unpublished.

[11] G.C. Fox and S. Wolfram, Phys. Lett. B 82 (1979) 134.

[12] Mark-II Collaboration, R. Jacobsen et al. Phys. Rev. Lett. 67 (1991) 3347.

[13] T. Sjöstrand, private communication.

[14] DELPHI Collaboration, P. Abreu et al., Nucl. Phys. B 421 (1994) 3.

[15] CDF Collaboration, F. Abe et al., Phys. Rev. Lett. 73 (1994) 225.

[16] G. Altarelli, "Status of precision tests of the electroweak theory"; preprint CERN-TH. 7319/94 (1994).

[17] See e.g. A.B. Lahanas and D.V. Nanopoulos, Phys. Rep. 145 (1987) 1.

[18] J. Bagger, S. Dimopoulos and E. Masso, Phys. Lett. 156B (1985) 357. 


\section{DELPHI}
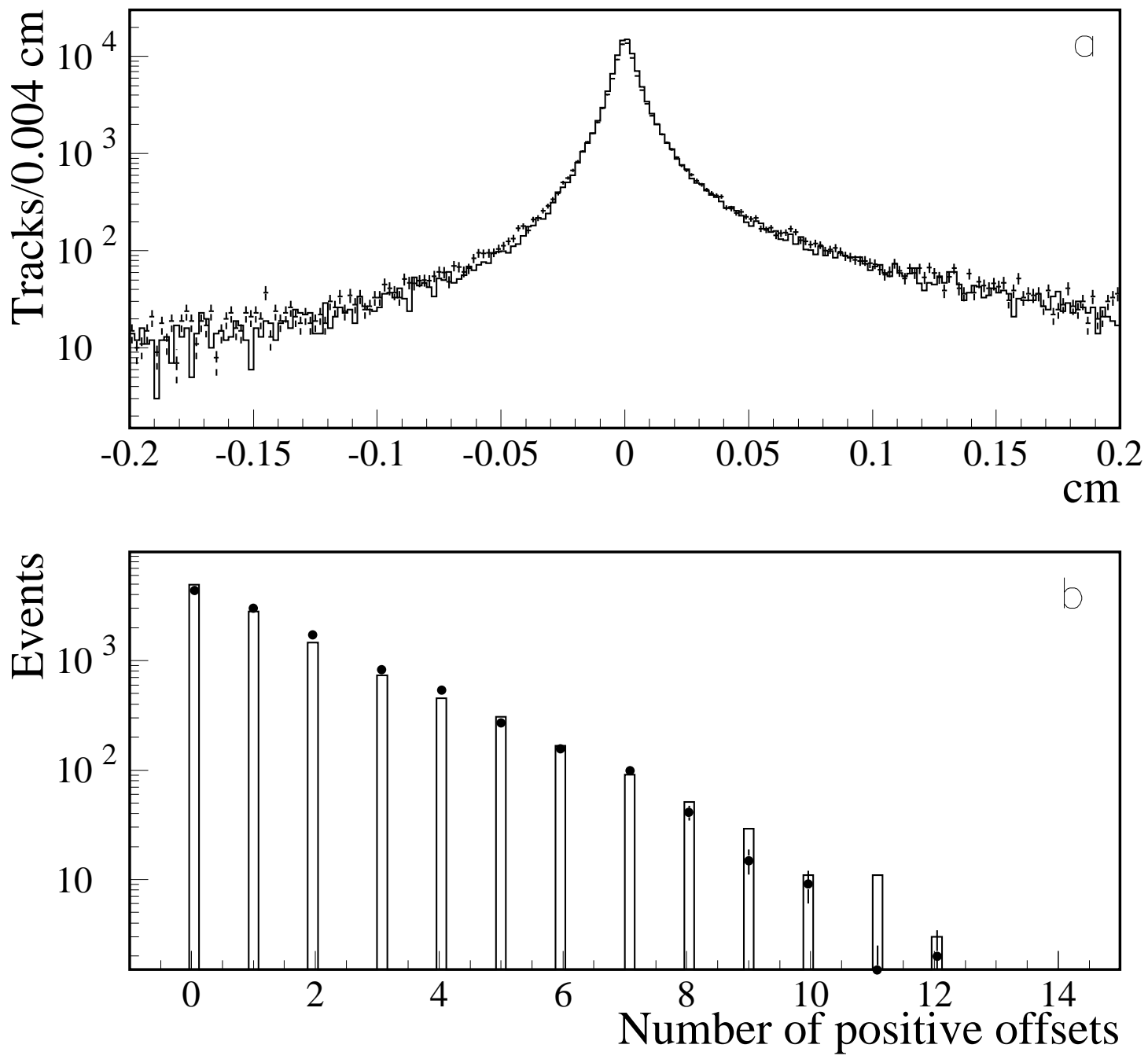

Figure 1: (a) The distribution of the impact parameter of accepted tracks in a sample of hadronic events. The solid line is the Monte Carlo prediction and the crosses represent the data. (b) The distribution of the number of positively signed impact parameters for the same sample. The rectangles represent the simulation and the dots the data. The number of tracks in the simulated events sample has been normalized to the one in the data. 


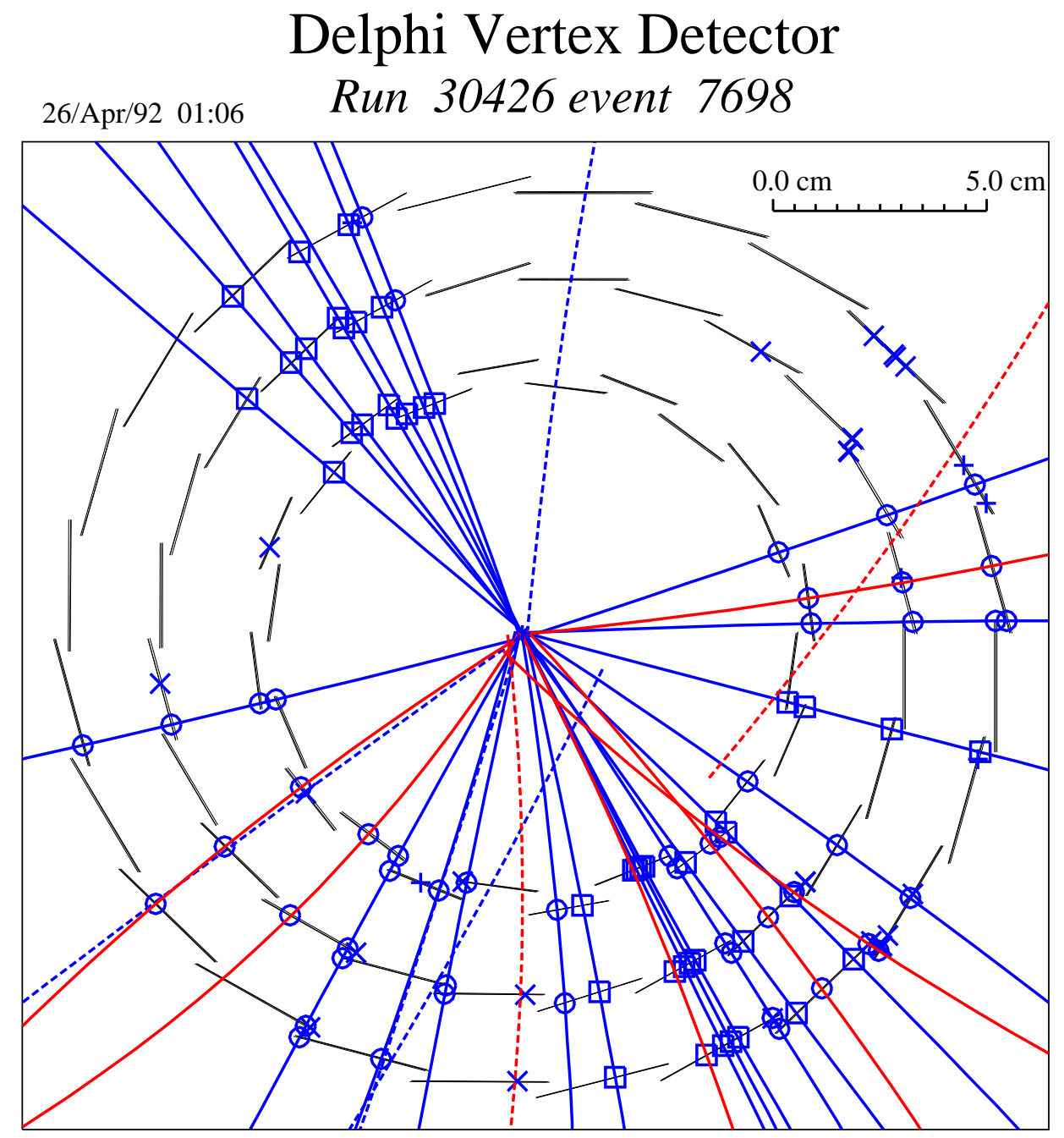

Figure 2: Display of a $4 \mathrm{~b}$ candidate showing the microvertex information. Dotted tracks had no associated VD hits (most of them are out of the VD acceptance). Circles (squares) represent $V D$ hits associated to tracks in the half detector with $z>0(z<0)$ and crosses hits which were not associated to tracks. 


\section{Delphi Vertex Detector}

26/Apr/92 01:06 Run 30426 event 7698

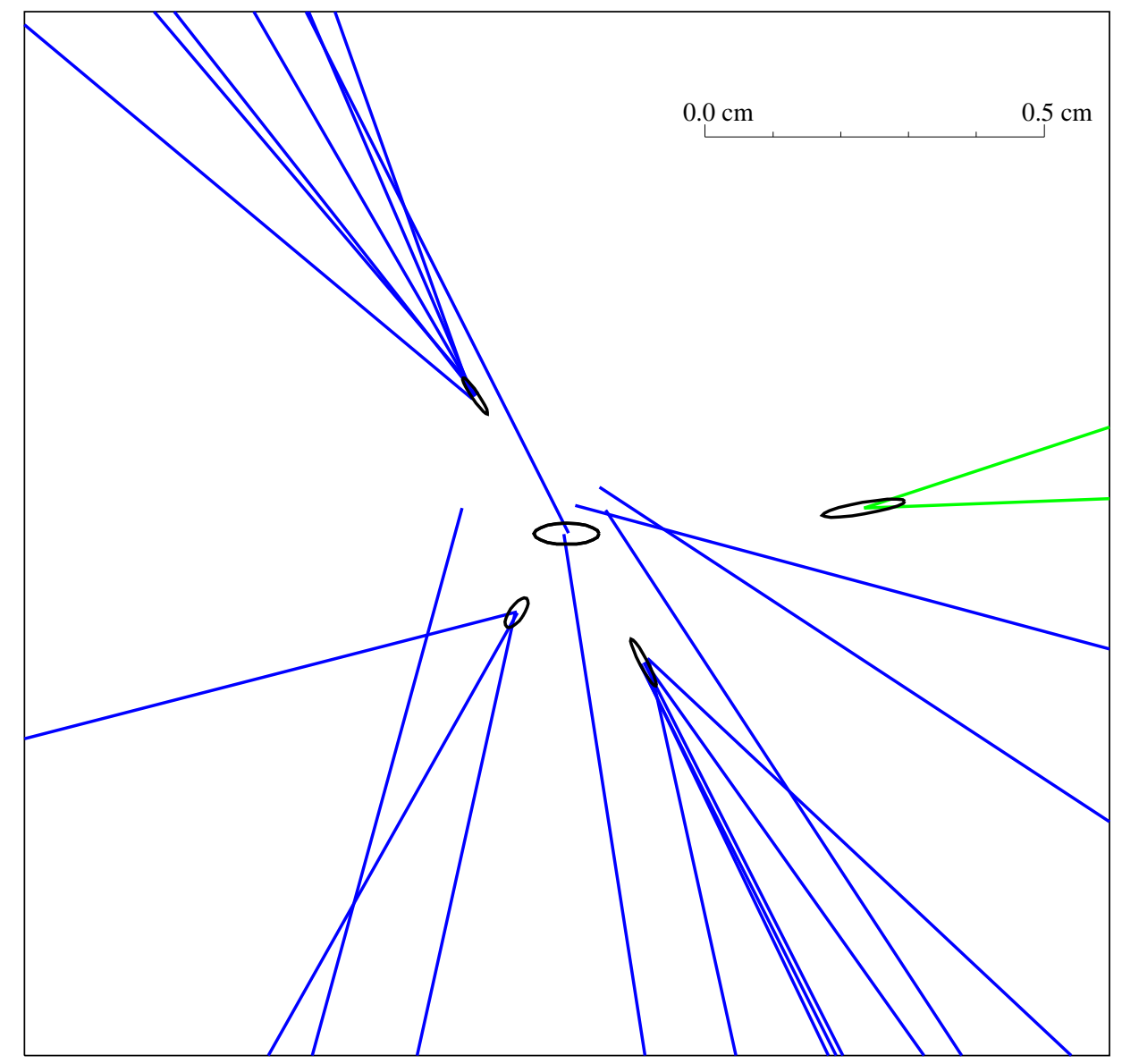

Figure 3: Display of the same candidate as in figure 2 on an expanded scale. Only tracks measured by the micro-vertex detector and with momenta above $0.5 \mathrm{GeV} / \mathrm{c}$ are displayed. The central ellipse indicates the beam position. Three displaced vertices have been reconstructed; the ellipses define the four-standard-deviation contours around them. $\mathrm{A} \mathrm{K}_{S}^{0}$ candidate is reconstructed on the right. 


\section{DELPHI}
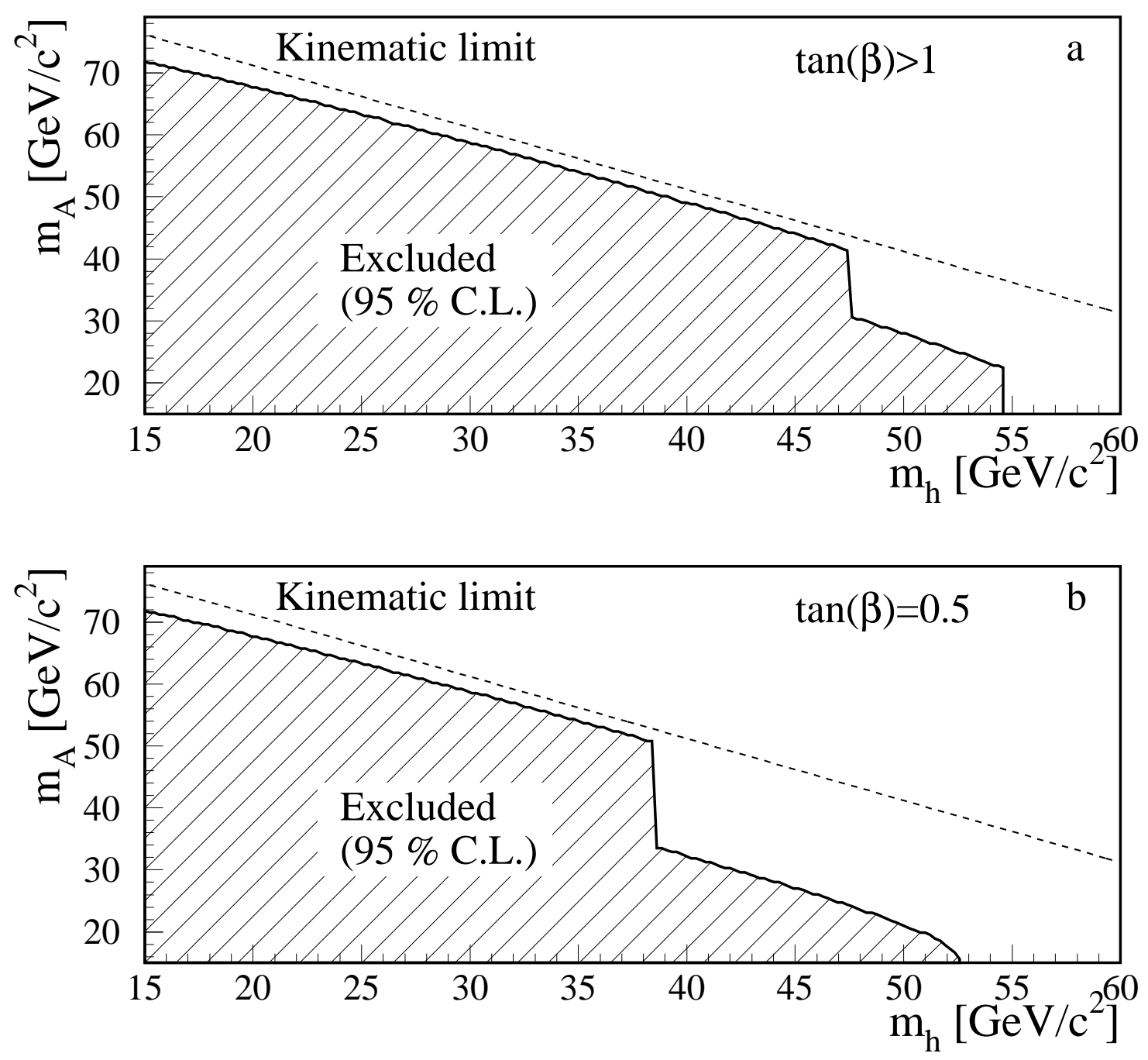

Figure 4: $\mathrm{m}_{A} / \mathrm{m}_{h}$ limit contour (at $95 \%$ confidence level) obtained from searches for $\mathrm{hA}$ and $\mathrm{hZ} \mathrm{Z}^{*}$ in the two-doublet case for $\tan \beta \geq 1$ and $\tan \beta=0.5$. The step in the limit corresponds to the transition to the regime where $h$ decouples from $b \bar{b}$. 


\section{DELPHI}

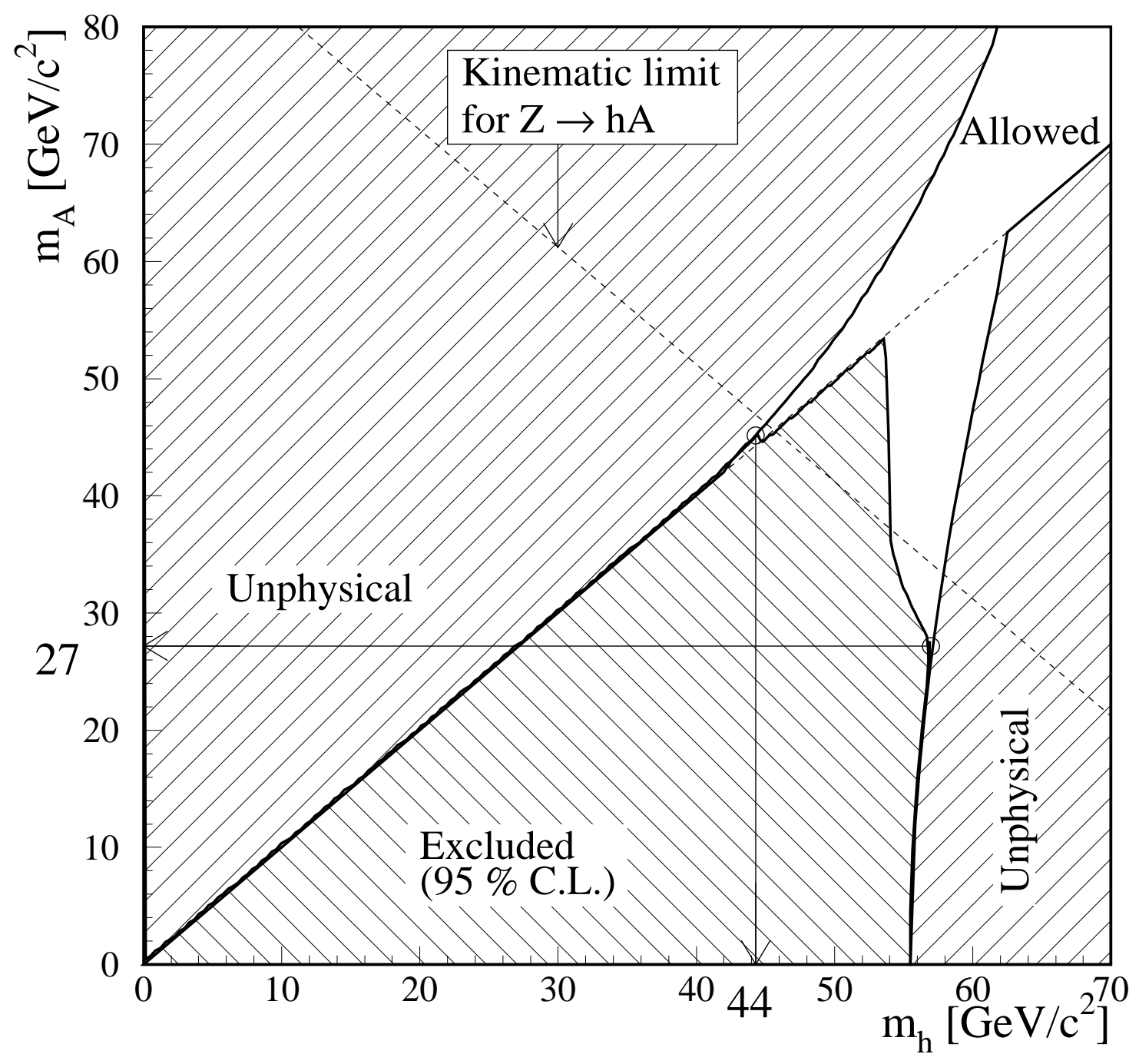

Figure 5: $\mathrm{m}_{A} / \mathrm{m}_{h}$ limit contour (at $95 \%$ confidence level) obtained from searches for hA and $\mathrm{hZ}^{*}$ channels in the MSSM case. A large fraction of the domain is not allowed by the model (the plot is obtained for $\tan \beta \geq 1$, assuming $\mathrm{m}_{t}=170 \mathrm{GeV} / \mathrm{c}^{2}$ and $\mathrm{m}_{s q}=1 \mathrm{TeV} / \mathrm{c}^{2}$ ). The one-loop formulae of reference [2] were used. 

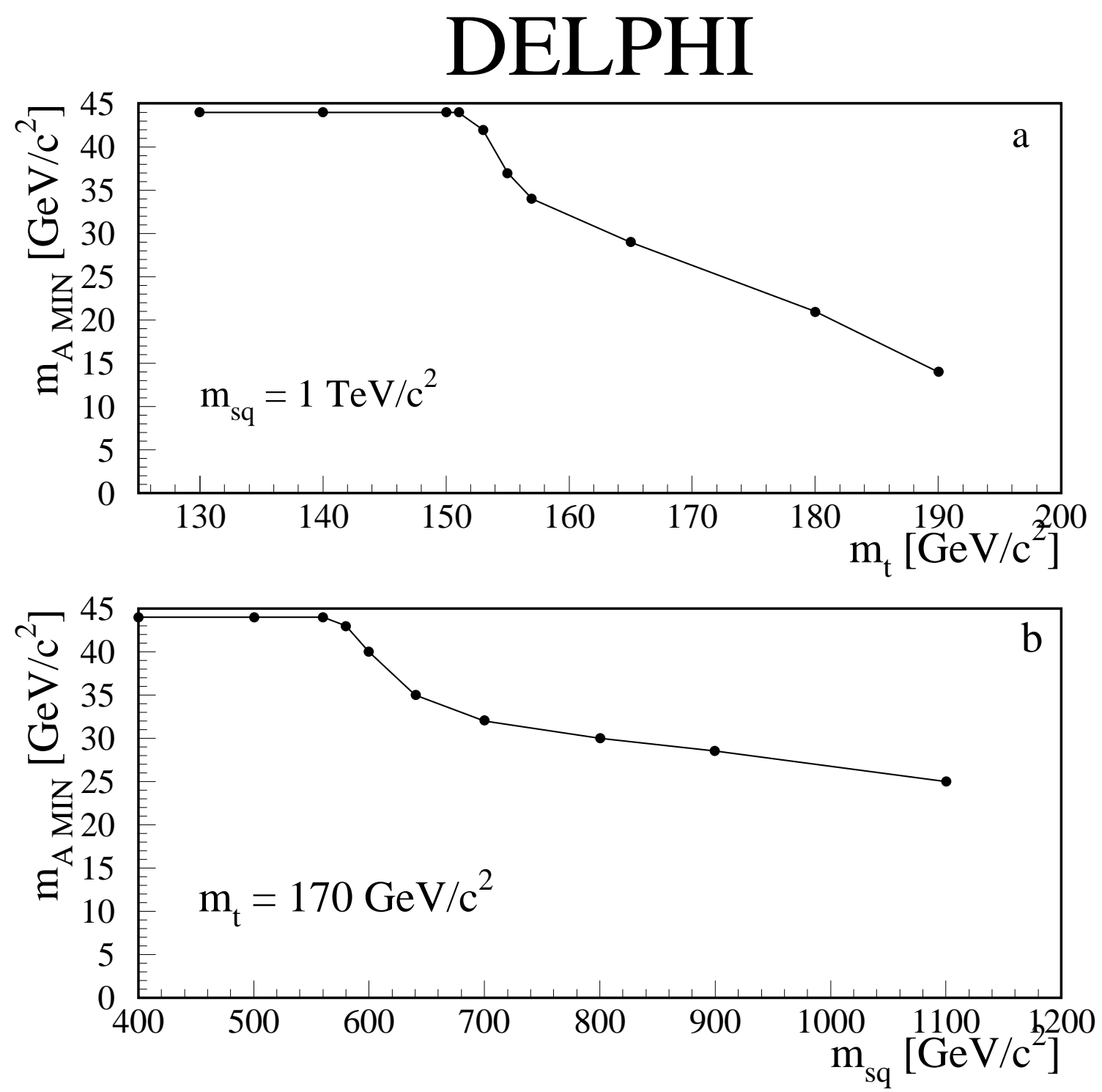

Figure 6: (a) the mass limit on $\mathrm{m}_{A}$ (at $95 \%$ confidence level) versus the top mass assuming $\mathrm{m}_{s q}=1 \mathrm{TeV} / \mathrm{c}^{2}$. (b) the mass limit on $\mathrm{m}_{A}$ (at $95 \%$ confidence level) versus the top-squark mass assuming $\mathrm{m}_{t}=170 \mathrm{GeV} / \mathrm{c}^{2}$. The one-loop formulae of reference [2] were used. 
DELPHI
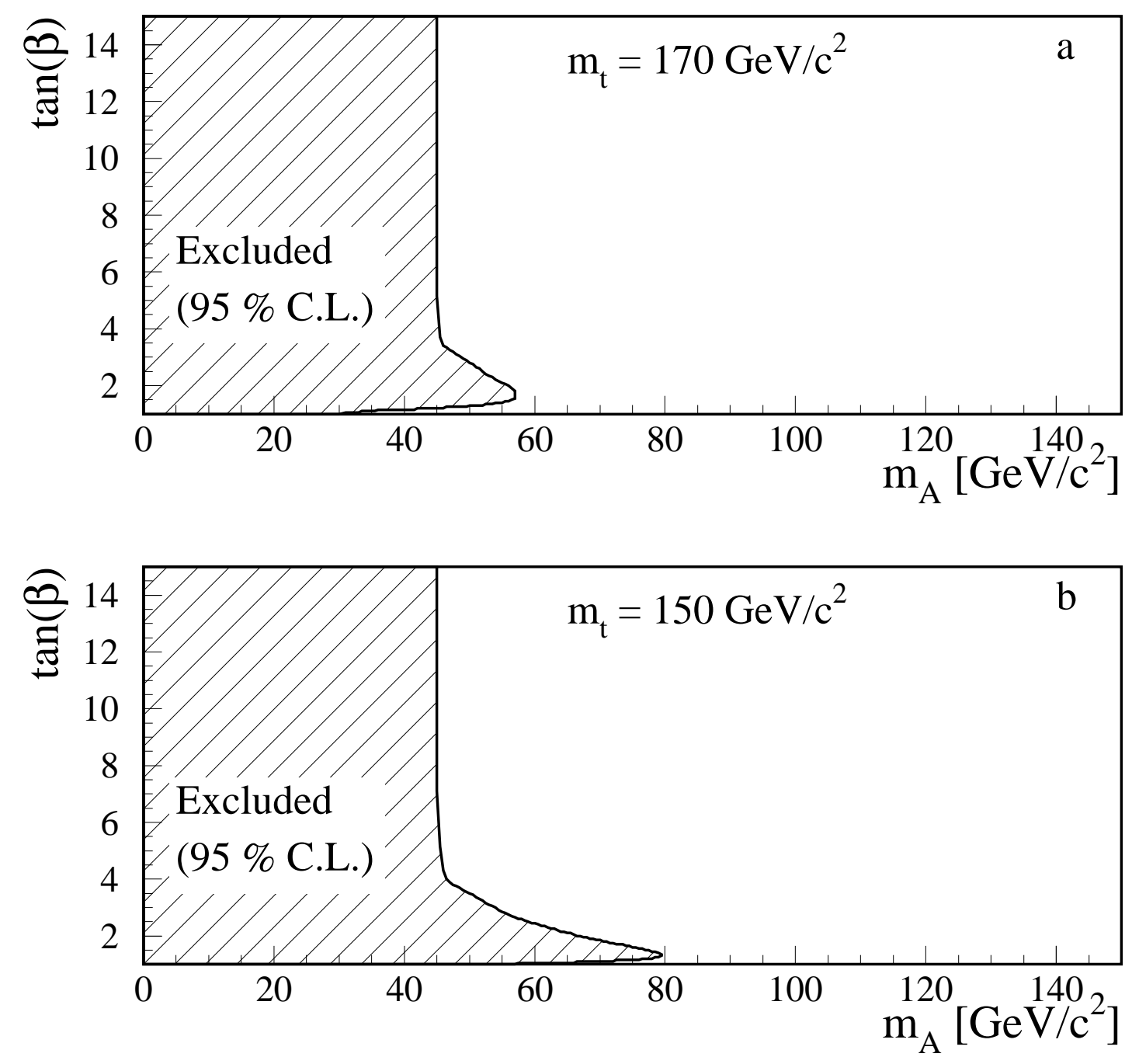

Figure 7: $\tan \beta / \mathrm{mA}$ limit contour (at $95 \%$ confidence level) obtained from searches for $\mathrm{hA}$ and $\mathrm{hZ}^{*}$ channels in the MSSM case with $\mathrm{m}_{s q}=1 \mathrm{TeV} / \mathrm{c}^{2}$ The two figures correspond to: (a) $\mathrm{m}_{t}=170 \mathrm{GeV} / \mathrm{c}^{2}$ and (b) $\mathrm{m}_{t}=150 \mathrm{GeV} / \mathrm{c}^{2}$. The one-loop formulae of reference [2] were used. 


\section{DELPHI}

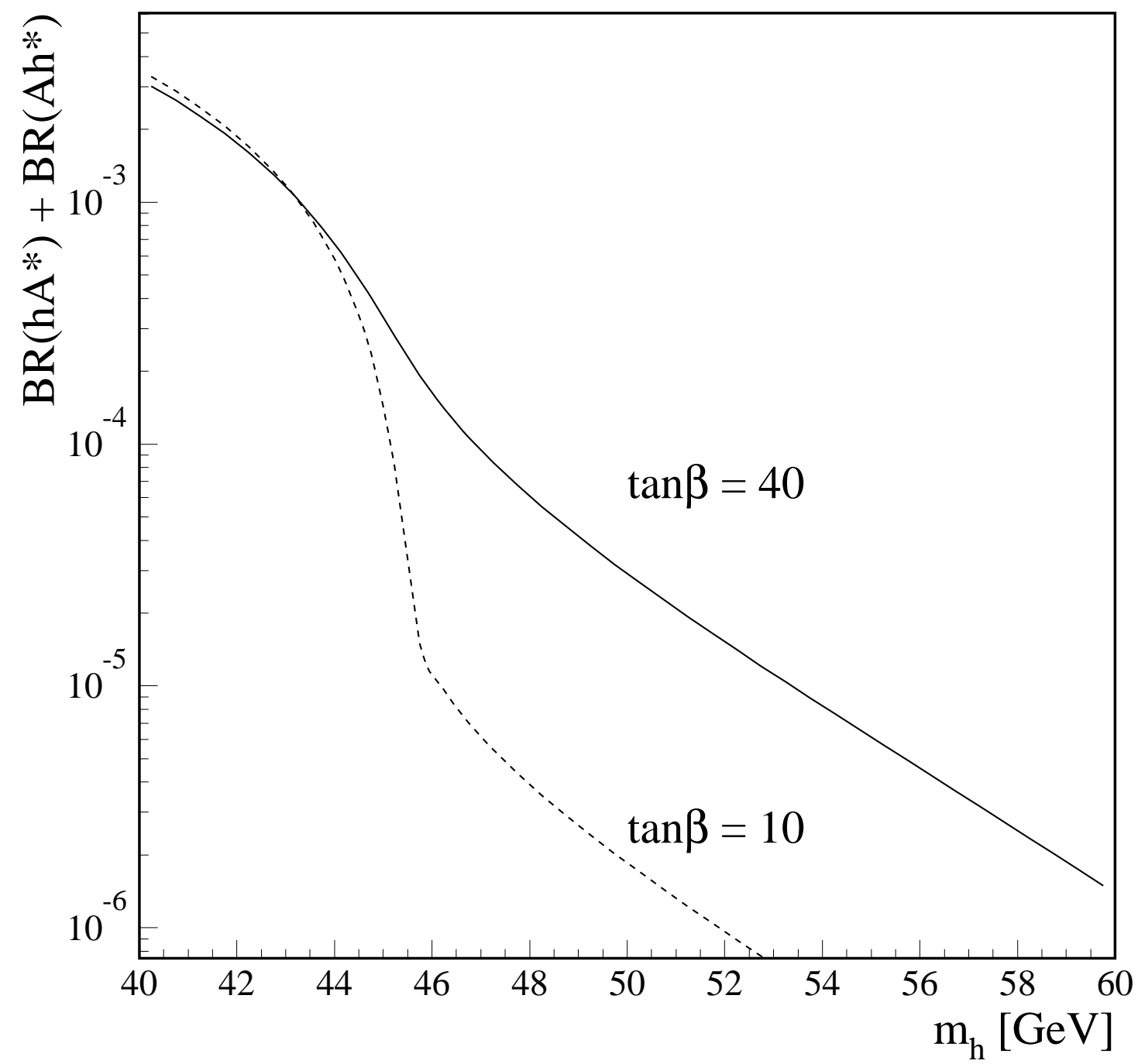

Figure 8: Results of the computation of $\mathrm{BR}\left(\mathrm{Z} \rightarrow \mathrm{hA} \mathrm{A}^{*}\right)$ given in the appendix. The full curve corresponds to $\tan \beta=40$, the dotted curve to $\tan \beta=10$, assuming that $\mathrm{h}$ and $\mathrm{A}$ have equal mass and that both $\mathrm{hA}^{*}$ and $\mathrm{h}^{*} \mathrm{~A}$ contribute. 